\title{
Explosive fragmentation criteria and velocities for vesicular magma
}

\author{
M.J. McGuinness ${ }^{\text {a,* }}$, B. Scheu ${ }^{\text {b }}$, A.C. Fowler ${ }^{c}$ \\ a School of Mathematics, Statistics and Operations Research, Victoria University of Wellington, New Zealand \\ b Earth and Environmental Sciences, LMU München, Germany \\ c MACSI, Department of Mathematics and Statistics, University of Limerick, Ireland
}

\section{A R T I C L E I N F O}

\section{Article history:}

Received 10 December 2011

Accepted 30 May 2012

Available online 7 June 2012

\section{Keywords:}

Explosive fragmentation

Mathematical model

Fragmentation velocity

Tensile strength

\begin{abstract}
A B S T R A C T
We present a new criterion for primary fragmentation of natural rock samples in shock-tube experiments, and new formulae for the effective strength of natural rock samples, for the size of primary fragments, and for fragmentation velocities. These formulae and the fragmentation criterion are given in terms of the physical properties of the rock and in terms of experimental parameters. The formulae and criterion are derived from numerical solutions and asymptotic analytic solutions to a novel recently published mathematical model for the explosive fragmentation of vesicular magma in shock-tube experiments. This model is singularly successful in accounting for the length-scales observed in these experiments. The criterion and formulae provide good matches to data from shock-tube experiments on natural samples.
\end{abstract}

(c) 2012 Elsevier B.V. All rights reserved.

\section{Introduction}

Explosive volcanic activity can take a wide range of forms, ranging from Hawaiian fire fountaining and Strombolian eruptions to highly energetic Vulcanian and Plinian eruptions. Fragmentation types may be roughly divided into two end-members depending on magma viscosity. In low-viscosity magma, bubbles can easily expand, ascend and coalesce, so that proposed fragmentation mechanisms include bursting bubbles and foam instability (Verhoogen, 1951; Sparks, 1978; Mangan and Cashman, 1996). On the other hand, in highviscosity magma, vesicles are not very mobile and bubble growth is constrained by viscous forces resulting in overpressurised vesicles. This magma tends to fragment in a brittle manner when the strength of the magma is exceeded, and this is usually taken to be due to the presence of pressurised vesicles (McBirney and Murase, 1970; Heiken and Wohletz, 1991; Gilbert and Sparks, 1998; Cashman et al., 2000). A common trigger mechanism for Vulcanian eruptions is the sudden unplugging of a sealed volcanic vent (Alidibirov and Dingwell, 1996; Yokoo and Ishihara, 2006; Mueller et al., 2008).

A number of experiments have been designed to explore in detail the fragmentation processes underlying explosive eruptions either using analogue material (Mader et al., 1994; Phillips et al., 1995; Ichihara et al., 2002; Namiki and Manga, 2005; Kameda et al., 2008) or natural samples (Alidibirov, 1994; Martel et al., 2000; Spieler et al., 2004b; Scheu et al., 2006, 2008). This study focusses on experiments

\footnotetext{
* Corresponding author. Tel./fax: +64 44635059.

E-mail address: Mark.McGuinness@vuw.ac.nz (MJ. McGuinness).
}

with natural samples where fragmentation was achieved by the sudden release of high-pressure gas in brittle magma. The experiments were conducted in an apparatus based on the shock-tube principle, built by (Alidibirov and Dingwell, 1996) and subsequently optimised and adapted (Spieler et al., 2004a; Scheu et al., 2008), yet still based on the same basic principles.

The setup consists of a high pressure section (autoclave, made from Nimonic ${ }^{\mathrm{TM}}$ stainless steel or acrylic glass) which is sealed off from a decompression chamber (a $3 \mathrm{~m}$ long steel tank of volume $0.38 \mathrm{~m}^{3}$ ) by a system of rupture discs. A cylindrical porous rock sample is tightly mounted in the autoclave. The autoclave is slowly pressurised by argon or nitrogen resulting in the pressurisation of the connected pore space of the rock sample. After pressure equilibration, and (where relevant) temperature equilibration, the high pressure section is rapidly decompressed by a systematic failure of the rupture discs. A shockwave propagates into the decompression chamber while a rarefaction wave travels down the autoclave towards the sample. When it reaches the sample, the pressure difference that develops in the pore gases may exceed the fracture strength of the surrounding rock, resulting in fragmentation of the sample, and ejection of the resulting detritus (Alidibirov and Dingwell, 1996; Spieler et al., 2004a; Scheu et al., 2006, 2008).

The experimental and mathematical results discussed here are derived from fragmentation experiments optimised for visual observation via high-speed video recordings (Scheu et al., 2008; Fowler et al., 2009). Autoclave and sample container are made from acrylic glass and special glue was used to fix the sample in the sample container. These experiments were pressurised using nitrogen gas. High-speed videos not only confirmed layer-by layer primary fragmentation events progressing inwards from the depressurised end of the sample, but also revealed secondary intra-block fracturing of primary fragmented layers. 
Further fragmentation speed experiments are also considered here (Kennedy et al., 2005; Scheu, 2005; Scheu et al., 2006; Mueller et al., 2008). They were also conducted at room temperature, but using argon gas and a steel autoclave allowing a broader range of applied pressures to be analysed. The samples are again glued into sample containers, but the glue is slightly stronger.

Sample and autoclave dimensions for both cases are very similar with samples being $24-26 \mathrm{~mm}$ in diameter and $40-60 \mathrm{~mm}$ in length. The autoclaves are both $200 \mathrm{~mm}$ in length and straight with the sample fixed slightly above the bottom end.

Different criteria based on physical models have been proposed for magma fragmentation (Verhoogen, 1951; McBirney and Murase, 1970; Wilson et al., 1980; Alidibirov, 1994; Zhang, 1999; Scheu et al., 2008); all of them provide insight into the fragmentation process, but none are able to describe or capture the entire phenomenon.

Recent analyses of fragmentation have attempted to serve as models for both erupting viscous magma and the laboratory experiments on natural samples discussed above. These models are based on fluid behaviour in the gas, the bubbly magma, and the fragmented rock/gas mixture (Alidibirov, 1994; Koyaguchi and Mitani, 2005; Koyaguchi et al., 2008). The resulting shock-tube model (Koyaguchi and Mitani, 2005; Koyaguchi et al., 2008) enforces conservation of mass, momentum, and energy as jump conditions across a gas rarefaction wave. The fragmentation surface is found to travel along a characteristic line with a constant fragmentation velocity. In that model, fragmentation is due either to sufficient overpressure in gas bubbles to exceed tensile strength, or to sufficient bubble volume to cause instability of thin magma foam. The time taken for the pressure to drop at the sample surface is not a consideration. The shock-tube modelling is in contrast to our work in two key respects - in that model the magma is assumed to be a fluid, and fragmentation is associated with closed porosity. In our model gas flows through a solid deformable porous medium, and changes in gas pressure are associated with gas flow through open porosity. This reflects our more narrowly focussed interest in modelling the laboratory experiments on solid natural samples.

With the particular aim of explaining the layered structure seen in experiments, Fowler et al. (2009) have constructed a new mathematical model from fundamental physical principles that explains layer-by-layer fragmentation. The idea is that as the rock column is depressurised, the stress in the solid relaxes very rapidly, while the gas pore pressure remains elevated, as its decline is reliant on escape of the gas through the pore space. If the permeability is low enough, or the decrease of the confining pressure is rapid enough, then the overpressure in the gas in the pore space may exceed the fracture strength, and it is this which causes the fractures in the model. Numerical solution of the model indicates that it can explain experimental results, both quantitatively and qualitatively.

Of particular interest is the manifestation of a length-scale in the primary fracturing event. Modelling prior to that of Fowler et al. (2009) does not predict such a length-scale. The time taken for gas pressure at the surface of a sample to drop by a significant amount, allows the pressure drop to effectively penetrate some distance into the sample, leading to the length-scale observed.

In this paper, we extend the results presented in Fowler et al. (2009), by considering their implications for the critical fragmentation pressure, effective sample strength, the length scale on which fragmentation occurs, and the speed of the primary fragmentation front. The dependence of these aspects of the fragmentation process on physical parameters like porosity, permeability, sample size and decompression speed is explored here.

In following sections of this paper, the mathematical model of Fowler et al. (2009) is summarised, detailed numerical results from this model are presented, a new criterion for fragmentation to occur is presented and compared with previous results, and the dependence of fragment size and fragmentation speed on physical parameters is explored.

\section{Fragmentation model}

The theory underlying the model of Fowler et al. (2009) is that of Biot $(1956,1962)$ which describes the poroelastic behaviour of the deforming rock as an elastic solid, coupled with the motion of the gas through the rock as a porous medium. As the flow is likely to be turbulent, Forchheimer's equation for interfacial drag is used instead of Darcy's law. A one-dimensional model of the solid deformation and gas flow is presented, in which the vertical coordinate from the base of the rock sample is $z$, and the principal variables of the model are the gas pressure $p$ and the solid displacement $w$.

For completeness, the model presented in Fowler et al. (2009) is summarised in dimensional terms here in Appendix A, where the conservation and stress-strain equations are written down. The rescaling that then leads to a reduced nondimensional set of equations is summarised in Appendix B. In the following sections, nondimensional variables are used without tildes on top, unless otherwise stated.

Nondimensional gas pressure $p$ is shown in Appendix B to satisfy the nonlinear diffusion Eq. (B.9), while solid displacement $w$ satisfies the force balance Eq. (B.10), which is a second-order boundary value problem. Before fracture occurs, the dimensionless height of the column is one, and the top surface is at $z=1$. On this top surface, we find that the pressure measured during shock-tube experiments in the gas chamber above the sample may be fitted after nondimensionalisation, with a decaying exponential of the form

$p=p_{c}=\exp (-a t)$

where $a$ is a dimensionless constant. It is related through the time rescaling to sample properties and to the experimental setup parameters as

$a=\frac{l}{t_{c} k^{1 / 4}} \sqrt{\frac{\phi \rho_{0} c_{F} l}{p_{0}}}$

where symbols are defined in Appendix B. In this expression, $t_{c}$ is a dimensional $e$-folding time for the surface pressure decay. This may vary with experimental setup. For the experiments we are considering, it is determined directly from measurements of the gas pressure at the surface of the sample to be of the order of $1 \mathrm{~ms} ; l$ is the column length, $k$ is the permeability of the sample, $\phi$ is the porosity, $\rho_{0}$ is the initial gas density, $c_{F}$ is an $O(1)$ coefficient in the Forchheimer drag expression, and $p_{0}$ is the initial confining pressure $(\mathrm{Pa})$.

Fracture occurs if the effective stress exceeds a critical value as given in Eq. (A.9). This can be rewritten as shown in Eq. (B.11) in terms of the strain $w_{z}$ as

$w_{z}>\frac{(1-\phi) \sigma_{Y}}{p_{0}} \equiv w_{z}^{\text {crit }}$ say,

where $\sigma_{Y}$ is the dimensional yield stress of the rock sample.

In the dimensionless model, before fragmentation, gas pressure decay depends on the adiabatic index $\gamma$, which is fixed once the gas is chosen, and on the decay rate $a$ at the sample surface. There are no other parameters in the diffusion equation governing gas pressure. Hence the formula for $a$ captures the essential dependence of gas pressure on rock and experimental parameters prior to fragmentation.

That there is essentially only one parameter (given $\gamma$ ) in the dimensionless pressure problem, Eq. (B.9) illustrates the power of the process of nondimensionalisation - dependence of pressure on all of the other parameters like permeability, porosity, sample length, and initial pressure, is contained in the parameters used to transform from dimensional to nondimensional variables in Eq. (B.1). The nondimensional problem is solved by some method, and solutions depend only on $a$ (given $\gamma$ ). Converting to dimensional values does not change the functional behaviour of solutions, it only changes 
the sizes of the variables. Dependence on the myriad of rock and experimental property parameters is revealed through the dependence of dimensionless $p, a, z$ and $t$ on these parameters, and through the dependence of dimensionless gas pressure $p$ on $a$.

The rock displacement depends on gas pressure, hence on $a$, and also on the glue parameter $\lambda$. Then the fragmentation criterion depends on the critical strain $w_{z}^{\text {crit }}$.

The effect of using different glue strengths is captured by varying the parameter $\lambda$. Numerical solutions to our model reveal that changing $\lambda$ has no effect on the critical pressure for fragmentation, or on the time $t^{*}$ taken to fragment. The fragment size is increased by reducing $\lambda$, by an amount roughly proportional to $\sqrt{\lambda}$, a scaling suggested by the form of the solid force balance $w_{z z}=p_{z}+\lambda_{w}$. Hence, fragmentation speed (the size divided by the time to fragment) is dependent on glue properties.

Indeed, if there is no glue, lift-off is immediate; if there is only glue at the base of the sample, the first fragmentation occurs there and the deduced fragmentation speed is faster than when glue is also on the sides of the sample, because fragment size is larger, as discussed in Fowler et al. (2009). If glue strength is too high, experimental experience is that the one-dimensional approximation used in our model fails, and fragmentation occurs by scalloping out the sample in a two-dimensional shape, leaving the glued sides attached to the apparatus. Experimenters also find that if the glue penetrates too deeply into the rock sample, altering the porosity there, this also gives problems (personal communication, $\mathrm{O}$. Spieler). It is clear that to hold the bulk of the sample in place initially without retaining the sides of it upon fragmentation, $\lambda$ must remain of order one, so cannot be expected to vary much in practice. Hence, our focus here is on the effect of the inherently more interesting parameters $a$ and $w_{z}^{\text {crit }}$, which take a range of values, as shown in Tables 1 and 2.

\subsection{Numerical results}

The model Eq. (B.9) for gas diffusion is solved using pdepe in Matlab, a partial differential equation solver that discretises in $z$ and then solves the resulting coupled ordinary differential equations in $t$, choosing the method and timestep dynamically according to the stiffness of these equations. Once the pressure is solved for, $w_{z}$ can be computed at any chosen time, by solving the ordinary differential Eq. (B.10). Since it is a linear boundary-value problem, a Green's function can be used to reduce the problem of finding $w_{z}$ to a simple quadrature, as shown in Appendix C.

Arguments are presented in Fowler et al. (2009) (and here illustrated using numerical solutions in Fig. 6) that as the pressure drops in the rock, the strain increases with a local maximum that penetrates deeper as it grows. Critical to this maximum being internal and not at the bottom of the rock sample, is the term $\lambda w$ in Eq. (B.10), which is due to the glue holding the sample in place. When the criterion for fragmentation is met, the glued rock sample is taken to immediately

Table 1

Ranges and typical values of the parameters that have significant ranges of values in the model, and the resulting values of $a$. Gas densities range from hot nitrogen $\left(35 \mathrm{~kg} \mathrm{~m}^{-3}\right.$ at $850^{\circ} \mathrm{C}$ ) to cold argon (140).

\begin{tabular}{lllll}
\hline Parameter & Min & Typical & Max & Units \\
\hline Permeability $k$ & $10^{-18}$ & $10^{-12}$ & $10^{-10}$ & $\mathrm{~m}^{2}$ \\
Initial pressure $p_{0}$ & 0.3 & 10 & 40 & $\mathrm{MPa}$ \\
Porosity $\phi$ & 0.05 & 0.4 & 0.9 & \\
Gas density $\rho_{0}$ & 35 & 115 & 140 & $\mathrm{~kg} \mathrm{~m}^{-3}$ \\
$a$ & 1.0 & 22 & 10,000 & \\
$w_{z}^{\text {crit }}$ & 0.003 & 0.04 & 3 & \\
$\lambda$ & 0.1 & 0.6 & 10 & \\
\hline
\end{tabular}

Table 2

Typical values of the physical constants of the model. The gas properties are those of nitrogen at room temperature.

\begin{tabular}{lll}
\hline Symbol & Meaning & Typical value \\
\hline$c_{F}$ & Ergun coefficient & 0.5 \\
$d_{g}$ & Glue thickness & $1 \mathrm{~mm}$ \\
$E$ & Elastic constant & $10^{11} \mathrm{~Pa}$ \\
$l$ & Sample length & $0.06 \mathrm{~m}$ \\
$R$ & Sample radius & $1.2 \mathrm{~cm}$ \\
$t_{c}$ & Chamber relaxation time & $1 \mathrm{~ms}$ \\
$\gamma$ & Specific heat ratio & 1.4 \\
$\eta_{f}$ & Gas viscosity & $1.8 \times 10^{-5} \mathrm{~Pa} \mathrm{~s}$ \\
$\mu_{\mathrm{g}}$ & Glue shear modulus & $2.5 \times 10^{9} \mathrm{~Pa}$ \\
$\rho_{s}$ & Solid density & $2.6 \times 10^{3} \mathrm{~kg} \mathrm{~m}^{-3}$ \\
$\sigma_{Y}$ & Yield stress & $1 \mathrm{MPa}$ \\
\hline
\end{tabular}

break (primary fragmentation) in numerical simulations, with an initial average gap between fragments set by the pore size and porosity. Strain at the gap faces immediately drops to zero. The broken piece of rock then accelerates upwards, driven by the pressure difference across it. Momentum conservation (Fowler et al., 2009) leads to the approximate nondimensional equation

$\frac{\epsilon}{\delta}(1-\phi) l_{i} \ddot{z}^{+}=\Delta p$

where $\Delta p$ is the pressure drop across the broken piece, and $z^{+}$is the location of the upper end of the gap. Gas conservation in the widening crack leads to the following equation for the nondimensional gas pressure $p$ in the gap,

$[z]_{-}^{+} \frac{d p^{1 / \gamma}}{d t}=\left[\phi\left|\frac{p^{1 / \gamma}}{p_{z}}\right|^{1 / 2} p_{z}-(1-\phi) p^{1 / \gamma} \dot{z}\right]_{-}^{+}$

where the notation [.] $]_{-}^{+}$means the difference between values at the upper and lower ends of the gap. The first term on the right-hand side of Eq. (4) is due to the gas flux from adjacent rock.

Solving the fragmentation problem now becomes a matter of solving these two ordinary differential equations simultaneously. They are coupled to the gas flux into the gap from adjacent rock, which must be obtained by solving the gas diffusion equation in each (remeshed) new fragment of rock. Small time steps are taken, so that the pressure in the gap can be incremented using Eq. (4), and the diffusion equation is solved in this time increment using the pressure profile from the previous time as initial condition, and the new pressure in the gap and at the upper surface as boundary conditions. Secondary fragmentation, of degassing moving fragments, also occurs in numerical simulations, and is observed experimentally (Scheu et al., 2008; Fowler et al., 2009).

This process is repeated, checking every piece of rock for further fragmentation at every time step, until pressures and strains drop to small values. Each fragmentation event leads to another pair of ordinary differential equations being added to the coupled set of equations to be solved. Contours of a typical resulting pressure history (Fig. 1) show pressures within the rock and in the gaps, dropping from the top downwards. The constant-in-space pressure regions mark the spreading gaps between accelerating fragments. Dips in pressure indicate fragmentation events.

A typical strain plot like that in Fig. 2 reveals more strikingly the fragmentation pattern, with strain rising to the critical value, dropping to zero there on fragmentation, and rising, possibly to the critical value, within the glued and the accelerating rock pieces as surface gas pressures fall further. The fragmentation history associated with these pressure and strain changes is presented in the first panel of Fig. 3, showing the location of fragment surfaces versus time. Note that 


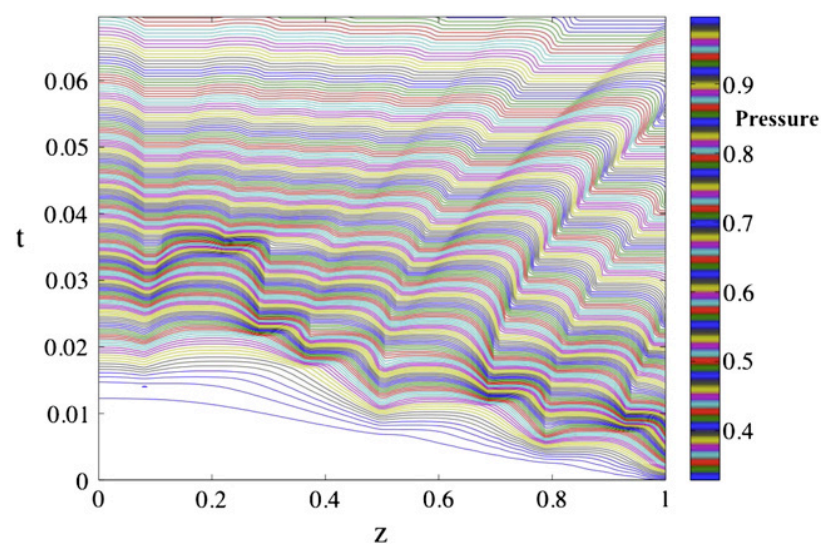

Fig. 1. Equally-spaced (in pressure) contours of constant pressure in rock fragments and in the gaps between, obtained from numerical model solutions, versus distance $z$ and time $t$. Pressure is set to drop at the rock surface at dimensionless $z=1$. Pieces of rock that fragment off are tracked and are included in the simulation. The (higher) gas pressures inside them and in the gaps between are also contoured. Broken pieces can be observed to accelerate to the right and exit from the graph at $z=1$. Gaps show up as horizontal pieces (constant in $z$ ). The colour key is on dimensionless pressure in the range of $0-1$. (For interpretation of the references to colour in this figure legend, the reader is referred to the web version of this article.)

individual fragments can be observed to accelerate away to the right (increasing $z$ ), as time increases, taking their strain history with them.

Of particular interest is a plot of the pressures observed in each of the gaps as seen in the second panel of Fig. 3, especially the pressure at the fragmentation front, at the face of the glued-down piece of rock. Small pressure oscillations may be observed in the gaps between fragments, associated with the kinematics as rock pieces come closer together and compress the gas between. The pressures in the gaps on average behave very similarly to the pressure at the uppermost surface, dropping at the same rate, delayed in time. This is because pressures in gaps rapidly adjust to the driving pressure drop in the chamber above the fragments - the kinematics is fast compared to diffusion inside the rock.

This is important for simplifying later calculations of fragmentation speed and timing, since the full problem with gas diffusion and accelerating fragments depends on a number of parameters $(a, \epsilon / \delta, \phi)$ as well as the size of each fragment, while the dimensionless problem of depressurisation of the glued-in piece depends only on the rate of pressure drop at the surface, which (for a given gas) depends only on the parameter $a$.

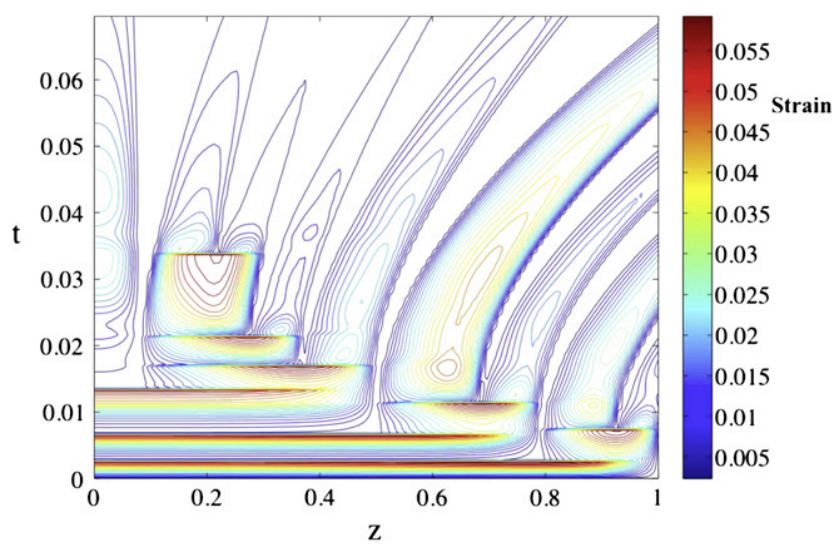

Fig. 2. Equally-spaced contours of constant strain $w_{z}$ (versus distance $z$ and time $t$ ), plotted from numerical solutions for fragmenting rocks and the gaps between. When maximum strain reaches the critical value (coloured red) the rock breaks there, and strain is set to zero at the new faces. (For interpretation of the references to colour in this figure legend, the reader is referred to the web version of this article.)
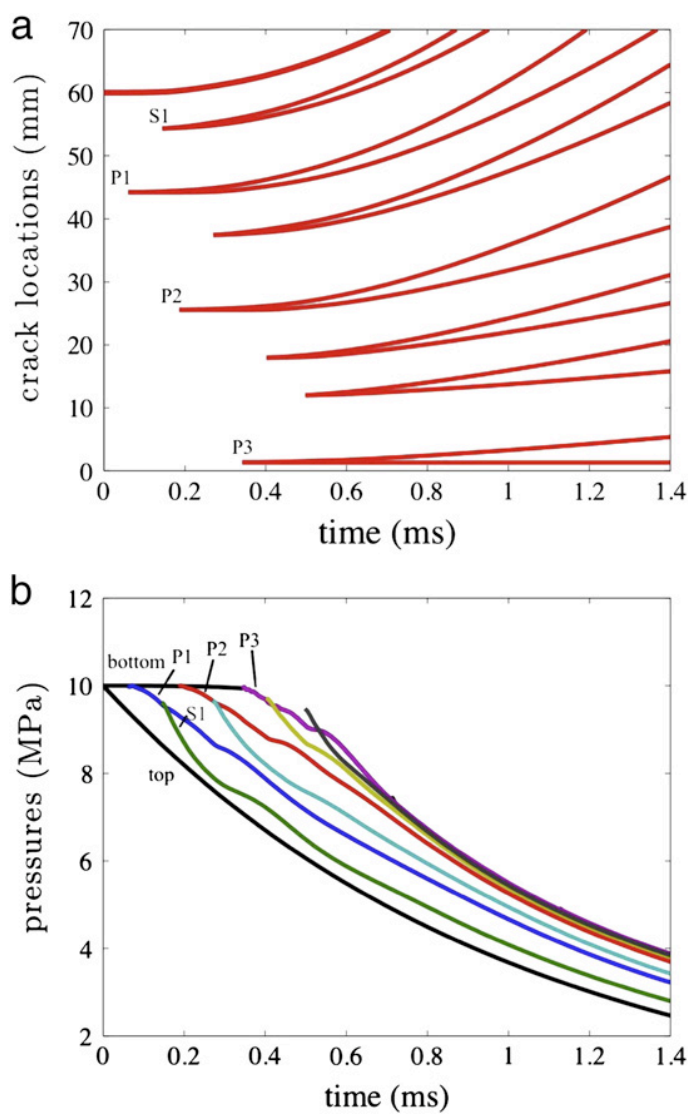

Fig. 3. (a) Fragment locations versus time, obtained by plotting the locus of the uppe and lower ends of any fragments obtained in numerical simulations; and (b) pressure versus time, at the surface of the rock sample (the lowest curve, labelled "top"), at its base (the highest curve, labelled "bottom"), and in the gaps between fragments. Primary fragment cracks are labelled in chronological order, P1, P2 and P3; the first secondary fragment crack is labelled "S1".

\section{Primary fragmentation}

Fragmentation in our model is caused by the tensile stress associated with pressure differences arising from gas discharge from the rock sample. The speed of decompression at the upper surface of the sample, relative to the speed with which gas can escape, together with the sizes of $p_{0}$ and tensile strength $\sigma_{Y}$, determines whether fragmentation will occur, when it occurs, and how big is the piece that breaks off.

Previous authors (Alidibirov, 1994; Koyaguchi and Mitani, 2005; Koyaguchi et al., 2008) have noted that in a shock-tube model (which treats the rock as a viscous fluid), the initial pressure $p_{0}$ and the porosity of the rock sample $\phi$ are important in determining whether a rock sample will fragment or not. This is based on energy arguments as well as empirical matching to fragmentation data.

In our model, changes in gas pressure are associated with gas flow through open porosity in a porous solid. The key role played by the parameter $a$ reflects the importance in our model of the speed of decompression in the gas rarefaction wave, which is slow compared to solid decompression.

We consider in particular two of the equations appearing in Koyaguchi et al. (2008), the first due to Alidibirov (1994) based on bubble-wall strength, which Koyaguchi et al. (2008) (Eq. (5)) found, gives the best fit to experimental data at both high temperatures $\left(850^{\circ} \mathrm{C}\right)$ and room temperature,

$p_{0}^{\text {crit }}=\frac{2 S_{a}\left(1-\phi^{1 / 3}\right)}{\phi^{1 / 3}}$, 
with fitted value of $S_{a} \approx 6.682 \mathrm{MPa}$; and the second a new criterion due to Koyaguchi et al. (2008) (Eq. (17)), which takes account of bubble-wall thickness,

$p_{0}^{\text {crit }}=\frac{2 S_{3}(1-\phi)}{3 \phi \sqrt{\phi^{-1 / 3}-1}}$.

The terms $S_{a}$ and $S_{3}$ are associated with the strength of the rock. A best fit to data for Eq. (6) gives $S_{3} \approx 2.18 \mathrm{MPa}$. Eqs. (5) and (6) define critical values of initial pressure to fragment rock of porosity $\phi$ and strength $S_{a}$ or $S_{3}$.

Although the modelling of Koyaguchi et al. (2008) is fundamentally different to that of Fowler et al. (2009), it is useful to recast our results into the same form as theirs for comparison with their results and with experimental data.

Two types of fragmentation are identified in our model (Fowler et al., 2009) and are seen in experiments, primary fragmentation where the advancing pressure front first breaks off pieces from the glued-in rock sample, and secondary fragmentation, where moving fragments subsequently break into smaller pieces.

The criterion for primary fragmentation has been written in terms of a critical strain as $w_{z} \geq w_{z}^{\text {crit }}$. We investigate the implications of numerical solutions to our model, for the dependence of the maximum value $w_{z}^{\max }$ of $w_{z}$ on the parameters $a$ and $\lambda$, to rewrite this criterion in a way that reveals its dependence on rock and experimental parameters. We also explore the time-dependence and location of fragmentation, when it does occur. Dependence on the parameter $a$ is paramount, since it contains the major rock and experimental parameters. Dependence on the glue parameter $\lambda$ is of less interest. In the remainder of this section, the usual value $\lambda=0.6$ is used.

\subsection{Primary fragmentation criterion}

Now we define an effective apparent material strength based on our modelling,

$S_{\text {model }}=\frac{\phi \sigma_{Y}}{w_{z}^{\text {crit }}}$,

so that our criterion for fragmentation is then that the initial pressure $p_{0}$ exceeds the critical value

$p_{0}^{\text {crit }}=\frac{(1-\phi) S_{\text {model }}}{\phi}$.

Note that in contrast to previous models, $S_{\text {model }}$, given by Eq. (7), is not a constant.

This is by design of similar form to Eqs. (5) and (6) above from Koyaguchi et al. (2008), and if $S_{\text {model }}$ was a constant, would have the same desirable properties of a vertical asymptote at $\phi=0$ and a zero at $\phi=1$. In general, as we will see, the form is not as simple as it appears here, since $S_{\text {model }}$ depends itself on $\phi$ and $p_{0}$, as well as on rock and experimental parameters, through $w_{z}$, so that $S_{\text {model }}$ is not a constant, and the form taken depends on the model results and upon other parameter values.

Numerical solutions of our model are used to find critical values of $p_{0}$, by replacing $w_{z}^{\text {crit }}$ in $S_{\text {model }}$ with the numerically found maximum $w_{x}^{\max }$ of $w_{z}$ over $z$ and $t$, for each value of the parameter $a$. This gives values for strength from numerical simulation,

$S_{\text {model }} \approx S_{m}^{\text {num }}=\frac{\phi \sigma_{Y}}{w_{z}^{\max }}$

we use varying values of the parameter $a$ to generate numerical values for $w_{z}^{\max }$ by solving numerically the nonlinear diffusion problem (Eq.
(B.9)). Given $a$ and $\phi$, a value for $p_{0}$ follows by rearranging the definition of $p_{0}$ (Eq. (2)) as

$p_{0}=B \frac{\phi}{a^{2}}$,

where

$B \equiv \frac{l^{3} \rho_{0} c_{F}}{t_{c}^{2} \sqrt{k}}$

Note that the value obtained for $p_{0}$ depends on $B$, which depends on a combination of rock and experimental properties, including gas density, sample length, sample permeability, and chamber depressurisation timescale.

We calculate from Eqs. (8) and (9)

$\sigma_{Y}=\frac{p_{0} w_{z}^{\max }}{1-\phi}$

which is the value for the strength $\sigma_{Y}$ of the natural rock sample that can be fragmented by a shock-tube experiment with the current values of $p_{0}$ and $\phi$, given $B$. This provides $\sigma_{Y}$ as a surface in $\left(p_{0}, \phi\right)$ space. This surface is presented in Fig. 4 as contours of constant strength, with $p_{0}$ and $\phi$ on the axes. Each contour may be interpreted as a numerical prediction of the critical initial pressure required to break a rock sample of porosity $\phi$ and tensile strength $\sigma_{Y}$, at the given fixed permeability.

Also plotted for comparison are the criterion from Koyaguchi et al. (2008), shown above as Eq. (6); the older curve Eq. (5) from Alidibirov (1994), as quoted in Koyaguchi et al. (2008) and using their best-fit value for strength $S_{a} \approx 6.682 \mathrm{MPa}$; experimental data from Koyaguchi et al. (2008) for natural samples at room temperature and at high temperature $\left(850^{\circ} \mathrm{C}\right)$; and experimental data from Mueller et al. (2008) exploring the effects of varying permeability on natural samples.

Three cases are plotted in Fig. 4, one for permeability $k=10^{-10} \mathrm{~m}^{2}$, one for $k=10^{-12} \mathrm{~m}^{2}$, and one for $k=10^{-14} \mathrm{~m}^{2}$. For all three cases, the rock samples that our numerical model predicts can be fragmented by $p_{0}$ values in the experimental range of 5-15 MPa, have values of tensile strength that sit in the range of 2-10 MPa, for larger values of porosity.

Our numerical model reproduces the important features of experimental behaviour, that the critical value of $p_{0}$ rises steeply as $\phi \rightarrow 0$, and approaches zero as $\phi \rightarrow 1$.

The effect of varying permeability on model results is also clearly illustrated in the sequence of plots in Fig. $4-$ as $k$ increases, so does the critical value of $p_{0}$. This effect is usually more obvious at higher porosities (Mueller et al., 2008), but is also evident in our results near small $\phi$ values, as the near-vertical contours are shifted sideways by this effect. One explanation for this dependence on permeability is that a higher permeability implies that gas can escape from the rock more easily, so that pressure gradients are smaller and pressure differences are smaller, so that there is less stress on the rock. Hence it is to be expected that it is more difficult to break higher permeability rock by this mechanism, so that intuition and experiment match our model results. Certainly, this is the observation from experiments on natural samples by Mueller et al. (2008). This is consistent with the observed shift to the right of our model contours at low porosity, or shift upwards at higher porosity, as $k$ increases. For the largest permeability case, the contour through $\sigma_{Y}=4 \mathrm{MPa}$ for our model is almost identical to the Alidibirov fit shown, which was considered to be the best fitting model in Eq. (5). The larger permeabilities provide a better match to experimental data, assuming that the tensile strengths of the natural samples lie in the range of 1-6 MPa.

Note that changing the gas density, sample length, or chamber depressurisation timescale would also affect the match to experimental data, by changing the value of $B$. 
Permeability and porosity (or vesicularity) are known to be interrelated, so we now consider what happens in our model if we allow permeability to vary with porosity. The Kozeny-Carman relationship is based on Poiseuille flow in capillary tubes, and can be written (Costa, 2006):

$k=C_{k c} \frac{\phi^{3}}{(1-\phi)^{2}}$,
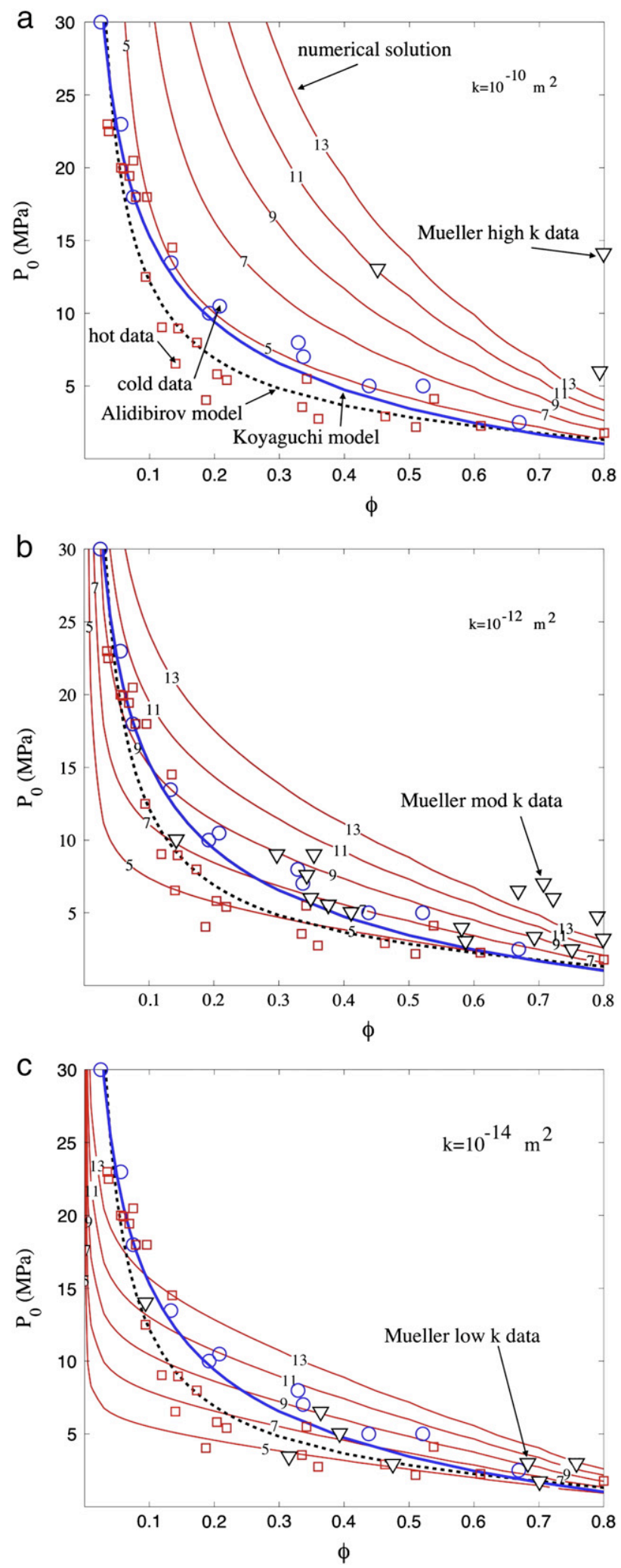

where $C_{k c}=c /\left(8 a_{v}^{2} \tau\right)$, where $c$ is an empirical geometric constant, $a_{v}$ is the specific internal surface area (the ratio of exposed surface area to solid volume), and $\tau$ is the tortuosity. However, the latter two parameters in general depend on porosity, so the overall dependence of permeability on porosity is not revealed by this relationship.

This has been adapted by Costa (2006) using a fractal formulation, with the intention of achieving a better match to measured relationships between permeability and porosity for volcanic vesicular rocks, to obtain the formula

$k_{\text {costa }}=C_{c} \frac{\phi^{m}}{(1-\phi)}$.

For vesicular basalts, Costa (2006) finds that the best fit parameters are $C_{c}=3.87 \times 10^{-12} \mathrm{~m}^{2}$ and $m=1.99$. For silicic pumices, he finds $C_{c}=1.46 \times 10^{-13} \mathrm{~m}^{2}$ and $m=1.47$.

We find that using the original parameter values of Costa (2006) for vesicular basalts and silicic pumices leads to poor fits to fragmentation data, and to permeabilities that are too low. Modifying the parameters to $C_{c}=1.5 \times 10^{-10} \mathrm{~m}^{2}$ and $m=0.5$ for example, leads to a better fit as illustrated in Fig. 5 . Note that this choice leads to a permeability range of $10^{-14}$ to $10^{-11}$, for a porosity range of $0.05-0.8$.

\subsection{Approximations to numerical simulations}

In this section we make some simplifications based on numerical solutions to our model. We seek to provide simple formulae, that summarise numerical results, and allow answers to questions about model behaviour without resorting to a full numerical computation.

As illustrated in Fig. 6, numerical simulations confirm that at early times, the location of $w_{z}^{\max }$ penetrates deeper into the rock sample, and the value of $w_{z}^{\max }$ increases as time increases. The occurrence of fragmentation is determined by whether this maximum reaches the critical value $w_{z}^{\text {crit }}$.

The numerically determined behaviour of $w_{z}^{\max }$ versus nondimensional time is given in Fig. 7 for a wide range of values of $a$. Note that for each $a, w_{z}$ increases to a unique maximum value $w_{z}^{\max }$ over both space and time, before decreasing again. All curves follow the same relaxing late-time behaviour, suggesting that degassing of the finite-sized rock sample begins to control the maximum strain at later times. We approximate this maximum value by using (Fowler et al., 2009),

$w_{z} \sim p-p_{c}, \quad \lambda \ll 1$

and we solve for pressure using a lumped-parameter approach. The average gas pressure $\hat{p}(t)$ in the rock sample drops because $p_{c}$ drops, but $\hat{p}(t)$ lags behind $p_{c}$. The maximum value of $w_{z}$ is then approximated by $\hat{p}$, taking $p_{c} \ll \hat{p}$. The gas diffusion equation may be averaged as in Appendix D to find the lumped-parameter analytic approximation

$\hat{p}=\frac{1}{\left(c_{1} t+1\right)^{c_{2}}}, c_{1} \equiv \frac{\gamma^{2}-1}{\sqrt{2} \gamma} \approx \frac{1}{2}, c_{2} \equiv \frac{2 \gamma}{\gamma-1}=7$.

This approximation $w_{z}^{\max } \approx \hat{p}$ is plotted as the dashed line in Fig. 7, and for all except the smallest values of $a$, it provides an excellent

Fig. 4. Numerical solutions of the coupled diffusion and stress equations have been used to calculate at what initial pressure $p_{0}$ a sample will fragment, versus porosity, given the tensile strength, and the permeability $k$. These numerical results are the thin contour lines, each labelled with a value of tensile strength in MPa. These may be compared with the data from cold (circles) and hot (boxes) experimental results as presented in Koyaguchi et al. (2008), with experimental data from natural samples of dome rock and pumice from Mueller et al. (2008) (triangles), and with two approximate formulae from Koyaguchi et al. (2008) (solid line) and Alidibirov (1994) (thick dashed line). The hot data is from Spieler et al. (2004b). Plot (a) is for $k=10^{-10} \mathrm{~m}^{-2}$, plot (b) is for $k=10^{-12} \mathrm{~m}^{-2}$, and plot (c) is for $k=10^{-14} \mathrm{~m}^{-2}$, in the numerical analysis. The Mueller et al. (2008) data is divided into three ranges of permeability, less than $10^{-13} \mathrm{~m}^{2}$ (low) is shown on the lower plot, between $10^{-13}$ and $10^{-11} \mathrm{~m}^{2}$ (moderate) is shown on the middle plot, and greater than $10^{-11} \mathrm{~m}^{2}$ (high) is shown on the upper plot. 


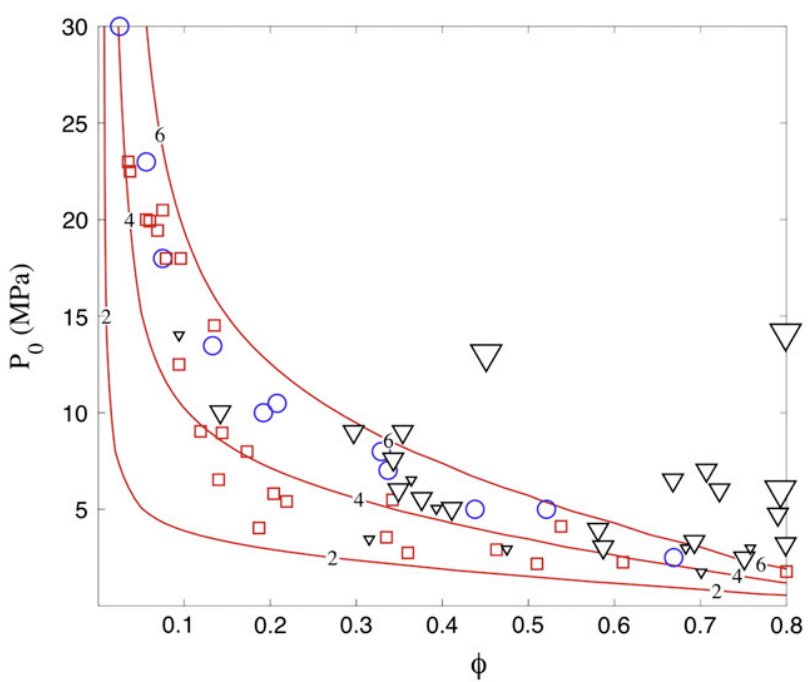

Fig. 5. Numerical solutions of the coupled diffusion and stress equations have been used to calculate at what initial pressure $p_{0}$ a sample will fragment, versus porosity, given the tensile strength. These numerical results are the contour lines, each labelled with a value of tensile strength in MPa. These may be compared with the data from cold (circles) and hot (boxes) experimental results as presented in Koyaguchi et al. (2008), and from experiments on natural samples by Mueller et al. (2008), whose data is divided into three ranges of permeability, less than $10^{-13} \mathrm{~m}^{2}$ (smallest triangles), between $10^{-13}$ and $10^{-11} \mathrm{~m}^{2}$ (medium sized triangles), and greater than $10^{-11} \mathrm{~m}^{2}$ (largest triangles). The hot data is from Spieler et al. (2004b). Permeability has been specified in terms of porosity, in a modified Carman-Kozeny approach. The model Eq. (11) proposed by Costa (2006) has been used with the values $C_{c}=1.5 \times 10^{-10} \mathrm{~m}^{2}$ and $m=0.5$.

match to numerical values of $w_{x}^{\max }$ (square symbols). For $a<2$, where the maximum is relatively broad, it falls below our approximation, because the assumption that $p_{c}$ vanishes faster than $\hat{p}$ fails to hold. This corresponds to the curve $w_{z}^{\max }(t)$ becoming vertical near $t=0.5$, near one end of our range of values of $a$.

We link $t$ and $a$ for both $w_{z}^{\max }$ and $\hat{p}$ by setting $t=1 / a$, the relaxation time for $p_{c}$, in Eq. (12). Hence fragmentation occurs when $\hat{p} \geq w_{z}^{\text {crit }}$

that is,

$p_{0} \geq p_{0}^{\text {crit }}=(1-\phi) \sigma_{Y}\left(\frac{1}{2 a}+1\right)^{7}$.

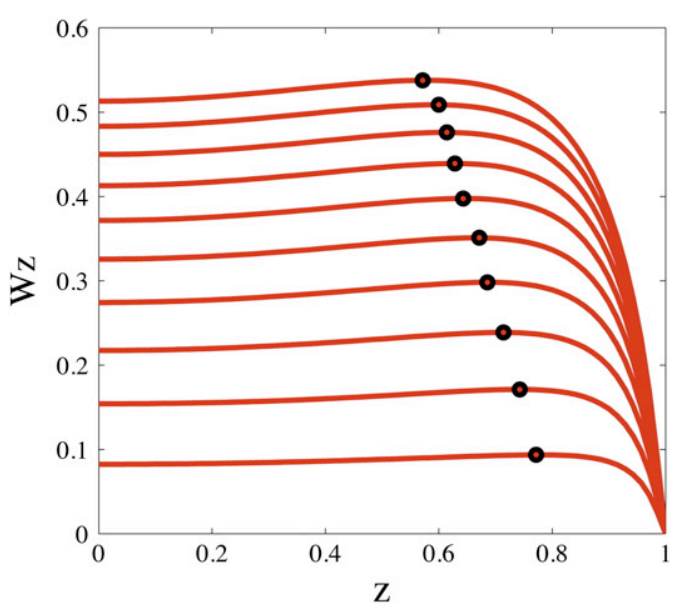

Fig. 6. The rock strain $w_{z}$ calculated from numerical solutions, plotted against $z$, for $a=22$ and ten dimensionless times stepping evenly from $1 / 220$ to $1 / 22$. The lowest curve is the earliest time $1 / 220$, and $w_{z}$ increases with time due to the decreasing pressure at the upper surface of the rock sample. The maximum value of $w_{z}$ at each time step is marked with a circle, and can be observed to penetrate deeper into the rock sample, and to increase, as time increases, at early times.

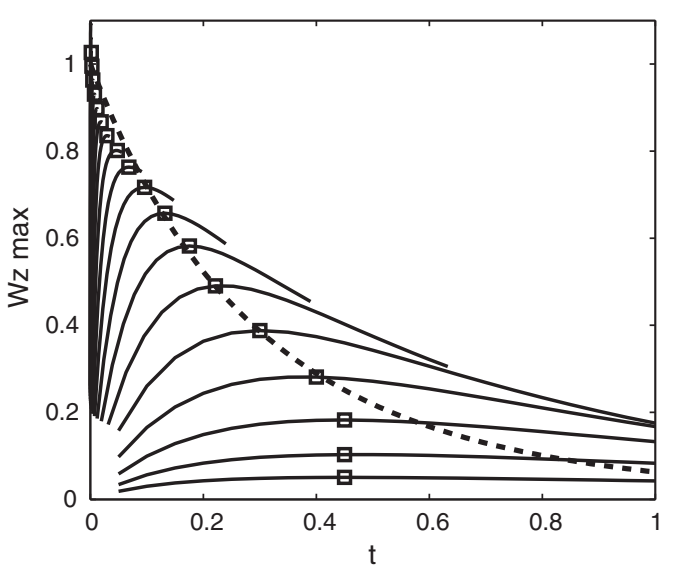

Fig. 7. The maximum value for $z \in(0,1)$ of rock strain $w_{z}$ calculated from numerical solutions, plotted against $t$, for $a=1$ stepping logarithmically in twenty steps to $a=10,000$ in order from the lowest solid curve to the upper-most. The squares mark the location on each curve of the maximum over time of the spatial maxima of $w_{z}$, and are highest for the largest values of $a$. The dashed curve shows the approximation Eq. (12) to $w_{z}^{\max }$ described in the text.

Then the $\log -\log$ plot of $w_{z}^{\max }$ versus $a$ in Fig. 8 shows that our lumped-parameter approximation $w_{z}^{\max } \approx \hat{p}$ provides a working fit to numerical values over four orders of magnitude of $a$.

A simpler piecewise linear approximation to the numerical results is also plotted in Fig. 8,

$w_{z}^{\max } \approx L=\left\{\begin{array}{cc}\frac{a}{10}, & 1 \leq a<10 \\ 1, & 10 \leq a \leq 10^{4}\end{array}\right.$,

and is of similar accuracy to the lumped-parameter result.

The lumped-parameter approximation to simulated results gives $\frac{\phi p_{0}^{\text {crit }}}{(1-\phi)}=S_{\text {model }}$, where $S_{\text {model }} \approx S_{m 1}=\phi \sigma_{Y} \hat{p}$,

while the simpler piecewise linear approximation gives

$S_{\text {model }} \approx S_{m^{2}}=\left\{\begin{array}{cc}\frac{10 \phi \sigma_{Y}}{a}, & 1 \leq a<10 \\ \phi \sigma_{Y}, & 10 \leq a \leq 10^{4}\end{array}\right.$

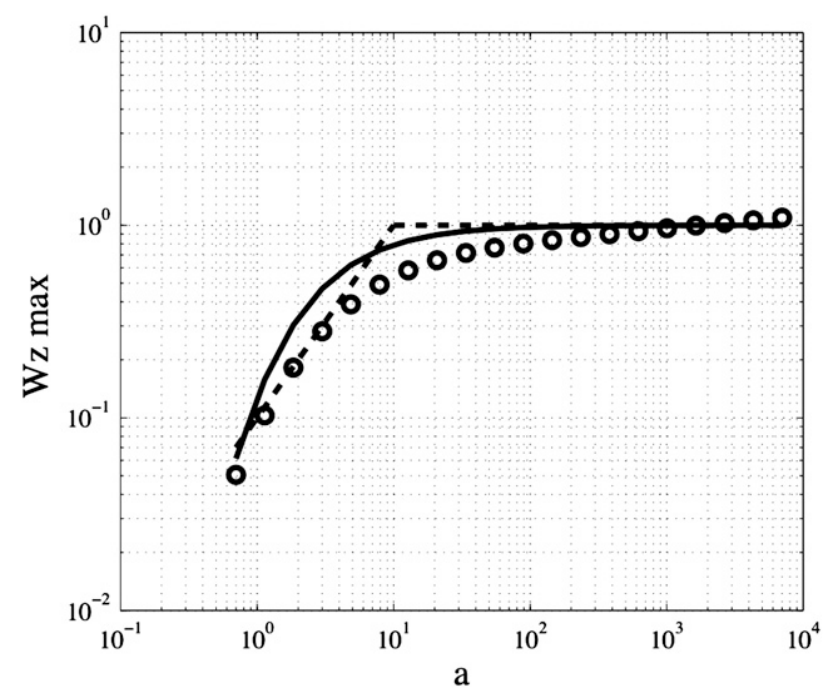

Fig. 8. Numerical values of $w_{z}^{\max }$ calculated from numerical solutions, plotted against $a$ (circles) using logarithmic scales. The solid line shows the lumped-parameter approximation Eq. (12) calculated in the text, with the substitution $t=1 / a$. The dashed line shows the simpler piecewise approximation Eq. (14). 
Note also that since $\hat{p}$ and $L$ depend on $a$, which depends in turn on $\phi$ and $p_{0}$ (see Eq. (2)), these relationships are implicit in relating $p_{0}$ and $\phi$. The simplest relationship, Eqs. (8) and (16), can be rearranged to obtain the critical pressure for fragmentation to occur, in explicit form:

$p_{0}^{\text {crit }}=\left\{\begin{array}{cl}\frac{100 \sigma_{Y}^{2}(1-\phi)^{2}}{B \phi}, & \frac{B \phi}{100(1-\phi)} \leq \sigma_{Y} \leq \frac{B \phi}{10(1-\phi)} \\ \sigma_{Y}(1-\phi), & \frac{B \phi}{10^{8}(1-\phi)} \leq \sigma_{Y} \leq \frac{B \phi}{100(1-\phi)}\end{array}\right.$

The form of $B$ in Eq. (10), and its appearance in Eq. (17), gives an approximation to the way that effective strength also depends on parameters $k, \rho_{0}, l$, and $t_{c}$.

This linear approximation is compared to experimental data and to the numerical simulation contours, for $k=10^{-12} \mathrm{~m}^{2}$, in Fig. 9 .

The approximation (Eq. (16)) suggests that fragmentation of rock specimens by gas could be used to measure the tensile strength of a wide range of rocks, provided that $a \geq 10$. Only the porosity is needed, and a series of experiments with increasing $p_{0}$ until the rock fragments at a critical value $p_{0}^{\text {crit }}$. Then from Eqs. (8) and (16), the tensile strength is given by

$\sigma_{Y}=\frac{p_{0}^{\text {crit }}}{1-\phi}$

Remarkably, this is independent of parameters like permeability, speed of depressurisation, sample length or gas density, provided only that $a \geq 10$. This is consistent with the experimental results of Mueller et al. (2008), that for low permeability (large $a$ ), there is no observed fragmentation dependence on sample permeability.

The plot in Fig. 9 of critical pressure versus porosity with constant permeability indicates that this formula for tensile strength is of the right form for all but the smallest porosities. It also suggests that the formula is only approximate, since for example at a porosity of 0.3 and a critical pressure of about $10 \mathrm{MPa}$, the formula would give a

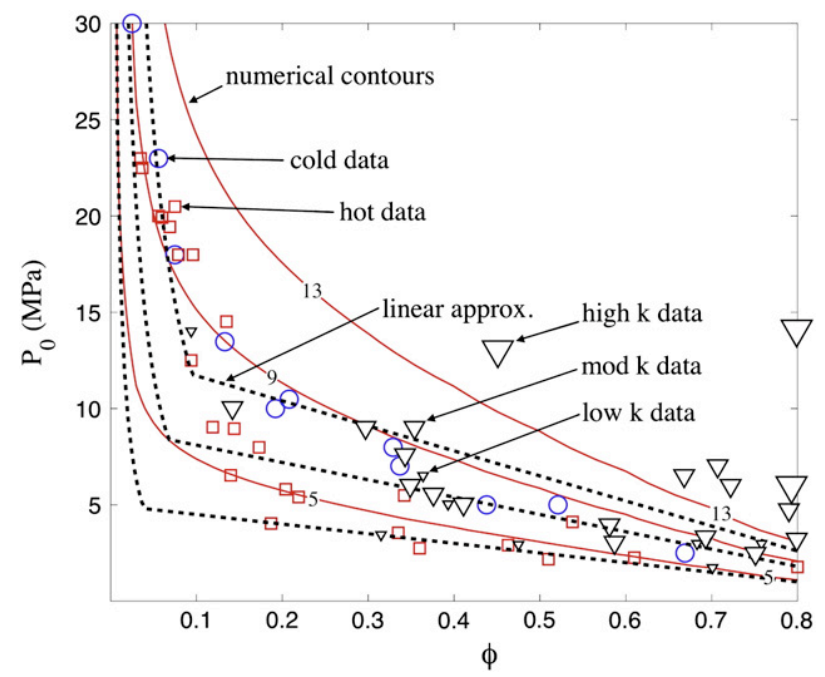

Fig. 9. Critical fragmentation pressure $p_{0}$ versus porosity $\phi$. Data from cold and hot experiments are shown as circles and boxes respectively. Data from experiments on natural samples of varying permeability by Mueller et al. (2008) is also shown, divided into three ranges of permeability, less than $10^{-13} \mathrm{~m}^{2}$ (smallest triangles), between $10^{-13}$ and $10^{-11} \mathrm{~m}^{2}$ (medium sized triangles), and greater than $10^{-11} \mathrm{~m}^{2}$ (largest triangles). The three solid lines are contours of accurate numerical simulations from our model, labelled with rock tensile strength $\sigma_{Y}=5,9$, and $13 \mathrm{MPa}$. The three dashed lines show the result of a piecewise linear approximation to effective strength, Eqs. (16) or (17) in the text, using the same values of $\sigma_{Y}$ as for the solid numerical contours. For the contours and linear approximations, a permeability of $10^{-12} \mathrm{~m}^{2}$ has been used, along with the other typical parameter values listed in Tables 1 and 2 . strength of $13 \mathrm{MPa}$ whereas the more accurate numerical solution says the strength is only $9 \mathrm{MPa}$, since the corresponding dashed and solid lines cross there.

Fig. 10 presents a similar picture of critical pressure versus porosity, but for the case that permeability is specified in terms of porosity, in a modified Carman-Kozeny approach. The model Eq. (11) proposed by Costa (2006) is used here, with the values $C_{c}=1.5 \times 10^{-10} \mathrm{~m}^{2}$ and $m=0.5$.

If rock and experimental parameter values give $1 \leq a \leq 10$, a more complicated formula results from Eqs. (8) and (16), and the tensile strength is then given by

$\sigma_{Y}^{2}=\frac{\phi p_{0}^{\text {crit }} l^{3} \rho_{0} c_{F}}{100(1-\phi)^{2} \sqrt{k} t_{c}^{2}}$

This formula compares well with that fitted by Mueller et al. (2008) to experimental results, where they found a linear dependence of the critical pressure on the square root of permeability, for large enough permeabilities.

The above results for the piecewise linear approximation to $S_{\text {model }}$ are echoed by the lumped parameter model, that for small $a$ the critical fragmentation pressure becomes independent of $a$, since the lumped parameter model criterion for fragmentation Eq. (13) can be written $p_{0} \geq p_{0}^{\text {crit }}=(1-\phi) \sigma_{y} f(a)$ where $f(a)$ is one for $a \ll 0.5$.

\subsection{Fragmentation time}

Assuming that fragmentation occurs after a short time compared to the time it takes for the average gas pressure to drop significantly, we now consider how long it takes for the first fragment to break off. This analysis is only valid then for $a>10$ and $p_{0}>\sigma_{Y}(1-\phi)$.

As above, we use the lumped-parameter pore gas pressure to approximate the criterion for breaking as

$w_{z} \approx \hat{p}(t)-p_{c}>w_{z}^{\text {crit }}$

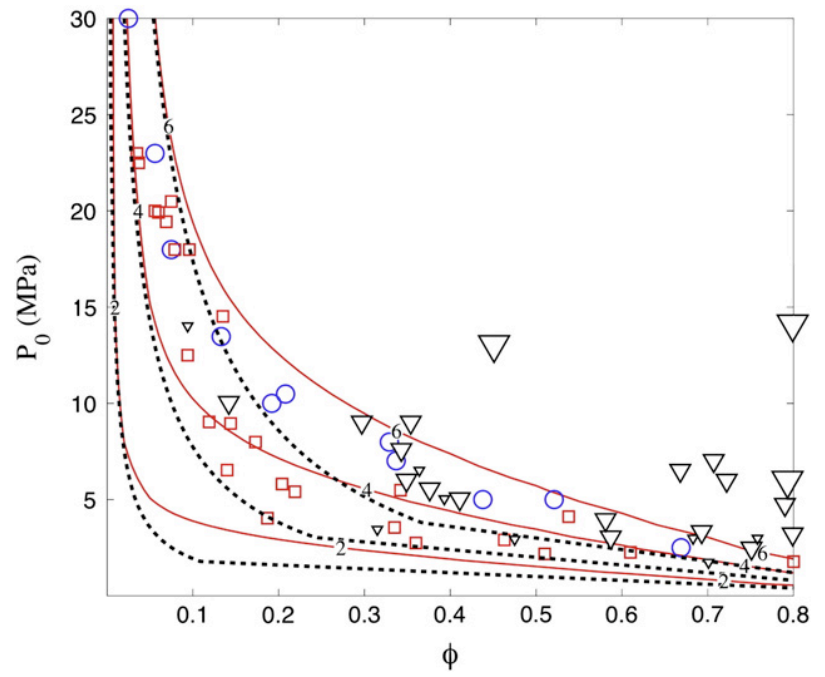

Fig. 10. Critical fragmentation pressure $p_{0}$ versus porosity $\phi$. As in Fig. 9, data from cold and hot experiments are shown as circles and boxes respectively, data from variable permeability experiments by Mueller et al. (2008) is shown as variable-sized triangles, and the three solid lines are contours of accurate numerical simulations from our model, but here labelled with rock tensile strength $\sigma_{Y}=2,4$, and $6 \mathrm{MPa}$. The three dashed lines show the result of a piecewise linear approximation to effective strength, Eqs. (16) or (17) in the text, using the same values $\sigma_{Y}=2,4$, and 6 MPa going from the lowest to the highest line. For the contours and linear approximations, permeability has been specified in terms of porosity, in a modified Carman-Kozeny approach. The model Eq. (11) proposed by Costa (2006) has been used with the values $C_{c}=1.5 \times 10^{-10} \mathrm{~m}^{2}$ and $m=0.5$. 
and we set $\hat{p} \approx 1$ for early times. The plot of $w_{z}^{\max }$ versus $1-p_{c}$ in Fig. 11 shows that $1-p_{c} \approx w_{z}^{\max }$ for large $p_{c}$ and $a>10$, in support of our approximation in Eq. (18).

Then, using $p_{c} \equiv e^{-a t}$, the inequality $1-p_{c}>w_{z}^{\text {crit }}$ is satisfied for times greater than the fragmentation time

$t^{*}=\frac{\left|\ln \left(1-w_{z}^{\mathrm{crit}}\right)\right|}{a}$.

Fig. 12 illustrates that this approximation gives a good match to fragmentation times obtained directly from numerical solutions of our model equations.

Also note that the dimensional time to fragmentation is given by

$t_{d}^{*}=t_{c}\left|\ln \left(1-w_{z}^{\mathrm{crit}}\right)\right|$,

which depends only on the (experimentally determined) timescale $t_{c}$ for gas depressurisation at the upper surface of the rock sample, and on rock porosity and tensile strength through $w_{z}^{\text {crit }}=(1-\phi) \sigma_{Y} / p_{0}$. It is independent of other rock properties like permeability and density.

\subsection{Fragment size}

A relationship between $z$ and $t$ will yield the location of the maximum of $w_{z}$, when $t=t^{*}$. We consider the similarity solution that approximates $p$ at early times (Appendix E), with $p$ being the solution of an ordinary differential equation that depends on the similarity variable

$\eta=\frac{1-z}{\beta_{2} t^{2 / 3}}, \quad \beta_{2}=\left(\frac{9 \gamma}{4}\right)^{1 / 3} \approx 1.5$

For problems with constant boundary conditions, the similarity variable provides a link between $z$ and $t$, for example in the case of linear diffusion problems giving the classic behaviour that $z$ varies as $\sqrt{t}$, related to the concept of depth of penetration. This leads to the hope that even in our nonlinear case with time-varying surface pressure, it might be the case that $w_{z}$ has a maximum at a unique value of the similarity variable $\eta$.

Numerical results in Fig. 13 indicate that $w_{z}^{\max }$ in fact varies with both $a$ and $\eta$, although it is of a similar order of magnitude for a wide range of values of the parameter $a$. However, for larger $a$ values, the

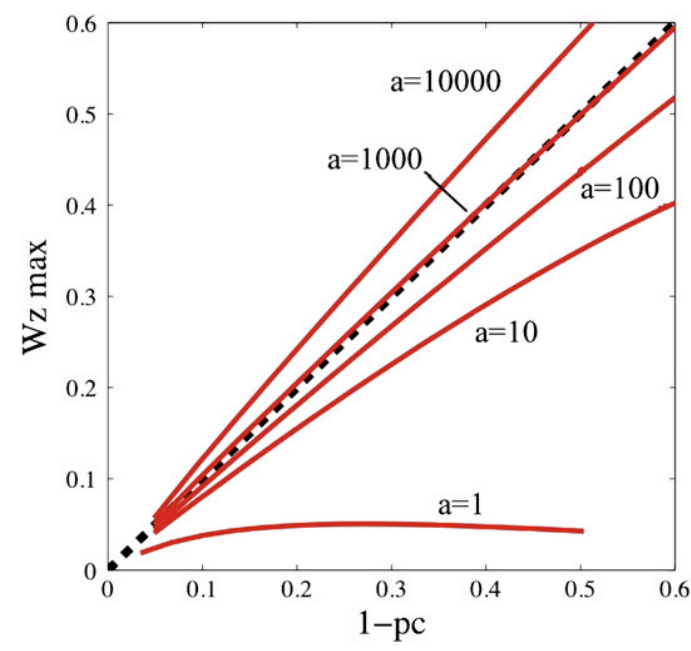

Fig. 11. The maximum strain $w_{z}^{\max }$ calculated from numerical solutions, plotted against the surface pressure drop $1-p_{c}$, for $a=10,000,1000,100,10$, and 1 (solid lines). The highest value of $a$ corresponds to the uppermost curve and the fastest surface pressure drop. As $a$ is reduced, $w_{z}^{\max }$ decreases. The dashed line shows where $w_{z}^{\max }$ exactly equals $1-p_{c}$, for comparison.

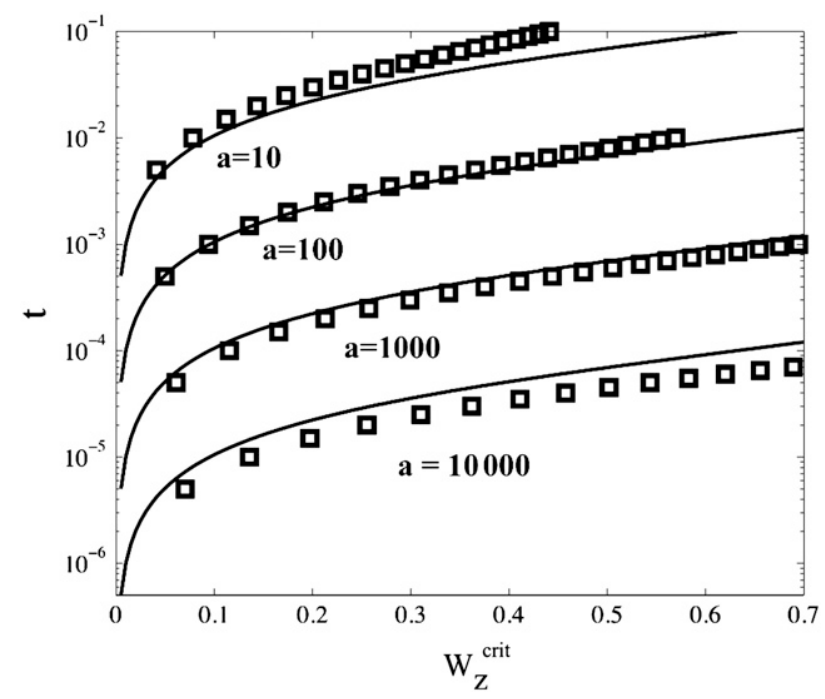

Fig. 12. A comparison of fragmentation times from numerical solutions of our model partial differential equations (boxes) with the simple approximation $t=\left|\ln \left(1-w_{z}^{\text {crit }}\right)\right| / a$, for $a$ values ranging from 10 to 10,000 . Note a log scale has been used to cover the wide range on the $t$-axis. The times are plotted against $w_{z}^{\text {crit }}$, which is the rescaled sample strength $(1-\phi) \sigma_{Y} / p_{0}$

dependence of $w_{z}^{\max }$ looks almost linear in $\ln a$, and also looks proportional to $1 / \eta$. A rough fit by eye to Fig. 13 suggests we can summarise the numerical simulations by the relationship

$w_{z}^{\max } \approx \frac{0.6 \ln a}{\eta}+0.01(\ln a-4.6)^{2}-0.25$

The fit of this relationship to numerical results is illustrated in Fig. 14, and seems satisfactory.

The size of the fragment $z^{*}$ that breaks off is found by setting $w_{z}^{\max }$ to $w_{z}^{\text {crit }}$, so that Eq. (20) becomes

$z^{*}=\frac{0.6 \beta_{2} t^{* 2 / 3} \ln a}{w_{z}^{\text {crit }}-0.01(\ln a-4.6)^{2}+0.25}$

The accuracy of this approximation is illustrated in Fig. 15 as a plot of $z^{*}$ versus $w_{z}^{\max }$ for numerical solutions (boxes) and for our approximation

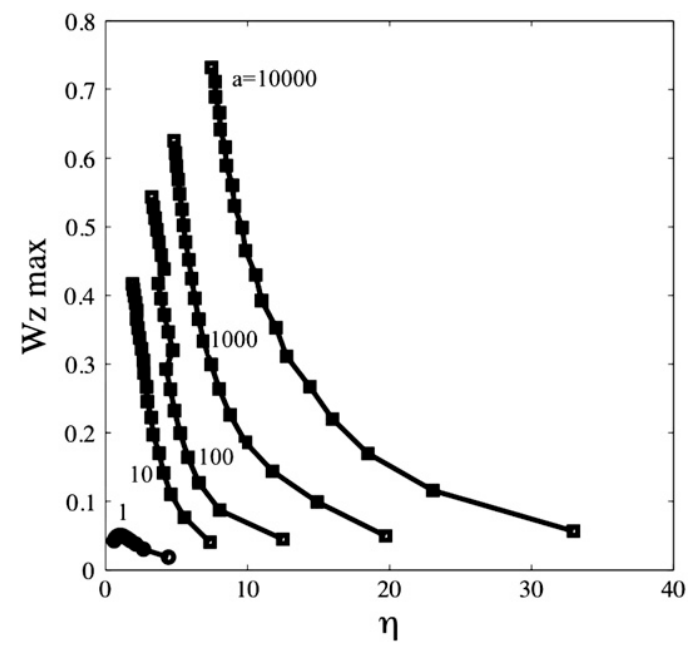

Fig. 13. Simulated values of the maximum rock strain $w_{z}^{\max }$, plotted against the similarity variable $\eta$, one curve for each of $a=10,000,1000,100,10$, and 1 , progressing from right to left through the curves. 
(curves), and in Fig. 16 as a plot of approximate versus accurate numerical $z^{*}$ values (circles, with the line illustrating a perfect match).

The dimensional size of the fragment is then

$z_{d}^{*}=0.9 l\left(\frac{\left|\ln \left(1-w_{z}^{\text {crit }}\right)\right|}{a}\right)^{2 / 3} \frac{\ln a}{w_{z}^{\text {crit }}-0.01(\ln a-4.6)^{2}+0.25}$,

which depends on all rock and gas parameters, through the parameters $a$ and $w_{z}^{\text {crit }}$.

\subsection{Fragmentation speed}

The fragmentation speed (the speed at which the fragmentation front penetrates from the top down into the glued stationary rock sample) is approximated by the speed of the first primary fragmentation event, relying on the numerical and experimental observation that primary fragmentation speeds appear to be constant. This corresponds to assuming that the kinematics are fast enough that the pressure drop at the surface of the remaining glued-in part of the sample is given by the experimentally imposed drop $p_{c}$, but delayed by the time taken for the fragmentation front to reach the current surface. Fig. 3 illustrates that numerical solutions to our model support this assumption - the pressure drops observed at each newly fragmented surface all look like translated-in-time versions of each other.

A sufficient condition for this to occur is that the movement of rock pieces in the fragmented region is rapid enough that decompression here continues as if the rock is suddenly not present, once it fragments. In this case, all terms in Eq. (4) and the equation preceding it may be equally important, but if the timescale for these equations is fast compared to gas diffusion in the remaining stationary piece of sample, this would be a sufficient condition. That is, if gas decompression in the fragmented region is much the same as gas decompression without rock present, this would be sufficient.

Note that we are also assuming here that the velocity of the decompression wave in the gas-fragment mixture is faster than the fragmentation speed controlled by gas diffusion in unfragmented rock. Such an assumption for the decompression wave in pure gas is integral to our model, and corresponds to our parameter $a$ being greater than one. This is true for the entire physical range of material

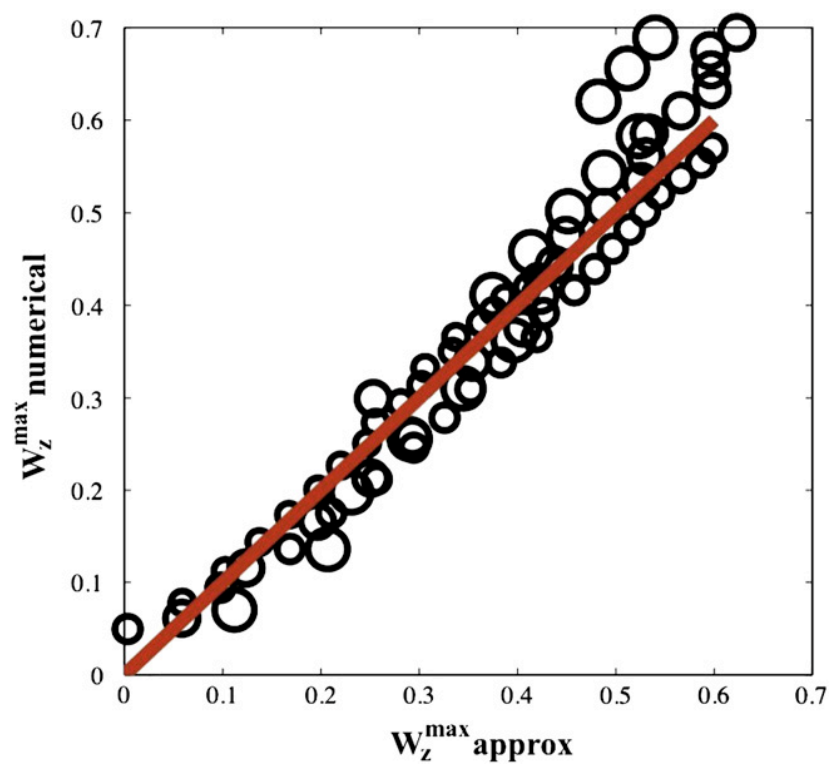

Fig. 14. The maximum rock strain $w_{z}^{\max }$ according to numerical model solutions, plotted against the approximate value $\frac{0.6 \ln a}{\eta}+0.01(\ln a-4.6)^{2}-0.25$ as circles, for early times and for $a=10,000,1000,100,10$, and 1 . The straight line illustrates perfect equality, and the size of the circles reflects the size of $a$ used.

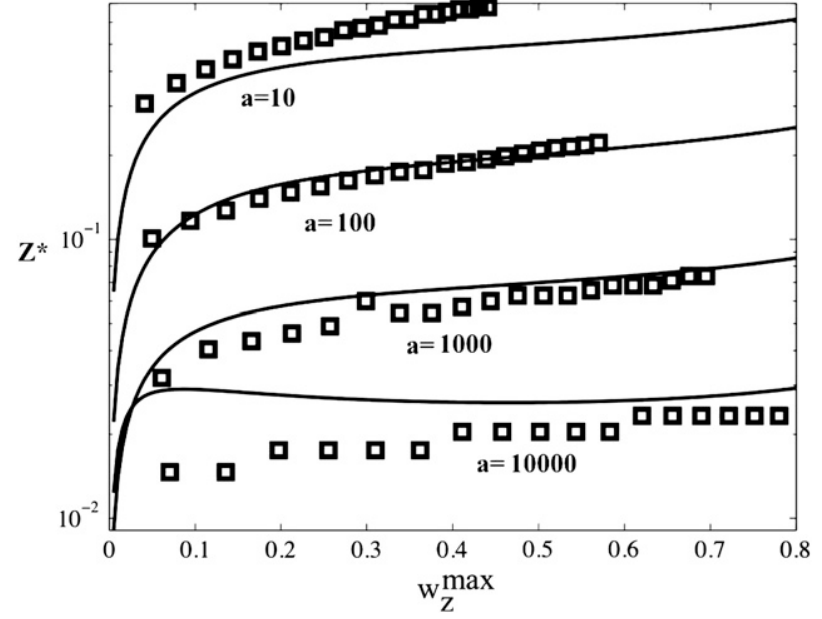

Fig. 15. The nondimensional size of the first fragment to break off, plotted against $w_{z}^{\text {crit }}$, for $a=10,100,1000$, and 10,000 . The boxes are the results of numerical solutions of the model equations, and the solid lines are the approximation Eq. (21).

properties considered in our work, see Table 1 . Then our sufficient condition above (that decompression in the gas/fragment mix is similar to decompression in pure gas) gives our assumption here. It also agrees with the results shown in Koyaguchi and Mitani (2005), Fig. 4b, where all fragmentation speeds are less than the speed of sound in the gas/fragment mixture.

Note that taking the first fragmentation event to give the speed of the primary fragmentation front, leads to the consequence that the fragmentation speed is constant in time.

The nondimensional speed of fragmentation $s^{*}$ then follows in terms of the parameter $a$ and the yield strain $w_{z}^{\text {crit }}$, by dividing the fragment size by the time to fragment, $s^{*}=\frac{z^{*}}{t^{*}}$, so that

$s^{*}=0.9\left(\frac{\left|\ln \left(1-w_{z}^{\text {crit }}\right)\right|}{a}\right)^{-\frac{1}{3}}\left(\frac{\ln a}{w_{z}^{\text {crit }}-0.01(\ln a-4.6)^{2}+0.25}\right)$.

A comparison of this approximate result with accurate numerical values for nondimensional fragmentation speed may be seen in Fig. 17 for various values of the parameter $a$.

In order to compare this approximation with data from shocktube experiments reported in Koyaguchi et al. (2008), we recast velocity as a function of the initial pressure $p_{0}$ and porosity $\phi$. We set

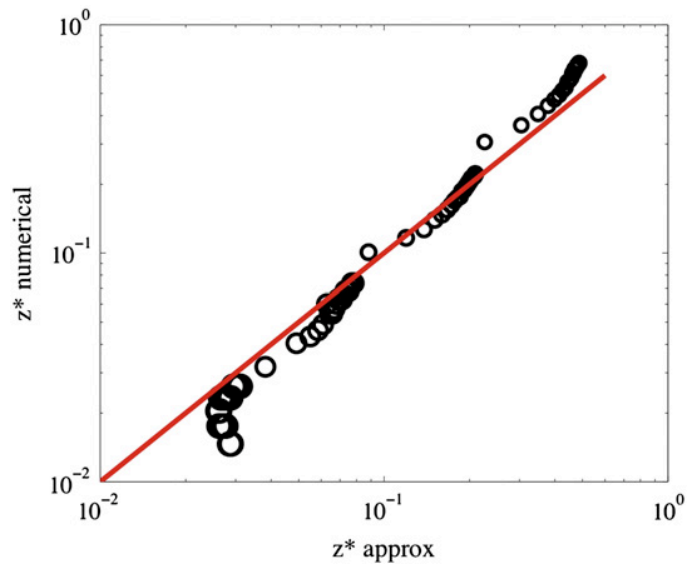

Fig. 16. A comparison of approximation Eq. (21) to numerical values, for the nondimensional size $z^{*}$ of the first fragment to break off, for $a=10,100,1000$, and 10,000 . The straight line illustrates perfect equality, and the size of the circles reflects the size of $a$ used - the larger the circle, the larger $a$ is. 
velocity to zero when $p_{0}$ is below the critical value for fragmentation, and we use the lumped-parameter approximation Eq. (13) to set this value. Then we convert back to dimensional velocity in $\mathrm{m}^{-\mathrm{s}^{-1}}$, to get

$v=\left\{\begin{aligned} 0, & p_{0}<(1-\phi) \sigma_{Y}\left(\frac{1}{2 a}+1\right)^{7} \\ s^{*} v_{0}, & \text { else }\end{aligned}\right.$

where $s *$ is given by Eq. (23), and

$w_{z}^{\text {crit }}=\frac{(1-\phi) \sigma_{Y}}{p_{0}}, \quad a^{2}=\frac{B \phi}{p_{0}}, \quad v_{0}=\sqrt{\frac{p_{0} l^{2}}{\phi B t_{c}^{2}}}, \quad B=\frac{l^{3} \rho_{0} c_{F}}{t_{c}^{2} \sqrt{k}}$.

This theoretical formula for fragmentation velocity is compared with and gives good matches to two different sets of data from shock-tube experiments, in Figs. 18 and 19, using tensile strengths of $14 \mathrm{MPa}$ for Unzen data and $13 \mathrm{MPa}$ for Montserrat data. The tensile strengths were chosen to give critical values of $p_{0}$ for fragmentation that matched the data values, so that the jumps from zero speed occur close to where the data indicates they should. The best match for both of these data sets for permeability-porosity relationships was obtained by using the Costa (2006) fractal formulation (Eq. (11)) of the Carman-Kozeny relationship with $C_{c}=4 \times 10^{-11} \mathrm{~m}^{2}$ and $m=4$. The resulting values for $a$ range from 8.3 to 883 for the Unzen case, and from 6.5 to 1422 for the Montserrat case.

With this choice, the model matches the experimental data within the natural scatter of data values.

The matches to fragmentation velocity from shock-tube theory illustrated in Koyaguchi et al. (2008) differ in appearance from our model in two respects - our curves are straighter, and we have a jump from zero fragmentation speed to a finite fragmentation speed at the critical pressure $p_{0}$. This jump is because just below the critical pressure, the maximum stress occurs at some finite depth and time, but is not yet enough to break the rock. Just above critical, this maximum is high enough to rupture the sample, and is still at some finite depth and time, hence gives the sudden appearance of a nonzero fragmentation velocity.

The above calculations of fragmentation time, size and speed are only for the first fragmentation event. The good match to fragmentation speed data from experiments suggests that this first event is a good indicator of overall fragmentation speed. After this event, the kinematics of the ejection of that fragment and subsequent fragments couple to the continued diffusion of gas in each piece of rock (Fowler et al., 2009) to modify the pressure changes at the faces of each piece. However, the kinematics is relatively fast. Our full numerical simulations (which do not make this assumption) indicate that,

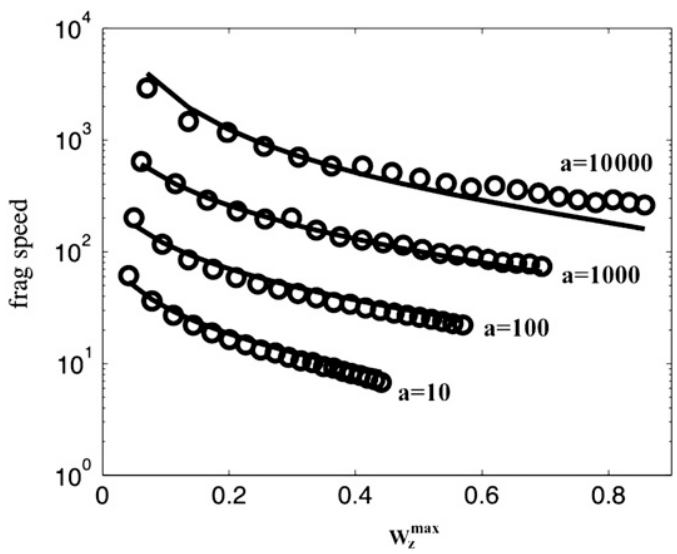

Fig. 17. Primary nondimensional fragmentation speeds versus $w_{z}^{\text {crit }}$ for $a=10,000$, 1000,100 , and 10. Circles show numerical simulation results, and solid lines show the approximate formula Eq. (23).

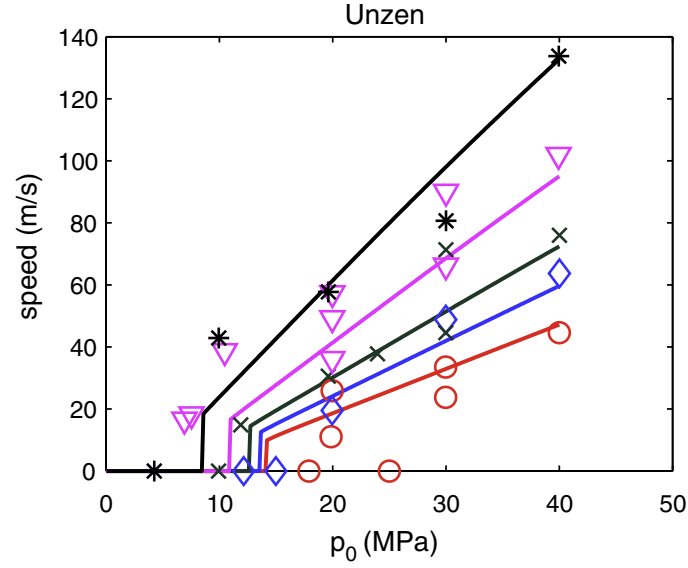

Fig. 18. Fragmentation speed versus initial gas pressure. The symbols are from shocktube experiments from Unzen (Scheu et al., 2006), with porosities of 0.061 (circles), 0.13 (diamonds), 0.21 (crosses), 0.34 (triangles), and 0.50 (asterisks). The lines are model predictions, using the same porosities, $\sigma_{Y}=14 \mathrm{MPa}$ and using the lumpedparameter approximation for maximum solid strain. Jumps to zero velocities occur at the modelled critical pressure for fragmentation to occur. Permeability is taken to vary with porosity as proposed by Costa (2006) in a fractal formulation of the Carman-Kozeny relationship, with the values $C_{c}=4 \times 10^{-11} \mathrm{~m}^{2}$ and $m=4$.

for example, the speed of fragmentation is almost constant as successive glued-in pieces break off (primary fragmentation), and the fragment sizes, while they decrease a little, remain of the same order. Furthermore, simulated pressures in the gaps between fragments can be observed in Fig. 3(b), after a brief initial lag, to decrease in a similar manner to the pressure at the upper surface of the fragmenting rock. Hence the first fragmentation event is an indicator of subsequent events, and has a similar velocity. Secondary fragmentation is also observed numerically and experimentally, where moving fragments themselves subsequently break in two, perhaps several times, due to internal gas pressures.

\section{Dependence on sample length}

Here we consider the implications of the results of previous sections, for the dependence of the fragmentation criterion (Eq. (17)) and the size of primary fragments (Eq. (22)) on the length of the

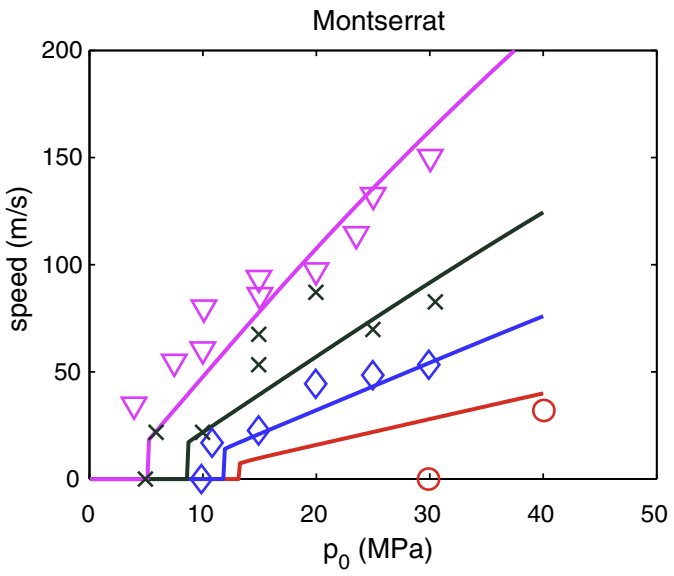

Fig. 19. Fragmentation speed versus initial gas pressure. The symbols are from shocktube experiments from Montserrat (Kennedy et al., 2005; Scheu, 2005), with porosities of 0.024 (circles), 0.20 (diamonds), 0.44 (crosses), and 0.67 (triangles). The lines are model predictions, using the same porosities, $\sigma_{Y}=13 \mathrm{MPa}$, and using the lumpedparameter approximation for maximum solid strain. Jumps to zero velocities occur at the modelled critical pressure for fragmentation to occur. Permeability is taken to vary with porosity as proposed by Costa (2006) in a fractal formulation of the Carman-Kozeny relationship, with the values $C_{c}=4 \times 10^{-11} \mathrm{~m}^{2}$ and $m=4$. 
sample $l$ (in $\mathrm{m}$ ). Since fragmentation time is independent of sample length, fragmentation velocity will have the same dependence on $l$ as primary fragment size.

The critical overpressure for fragmentation, in expression Eq. (17), switches from a simple dependence solely on effective tensile strength $\sigma_{Y}(1-\phi)$ to a more complicated dependence on rock and experimental properties, as the tensile strength increases through the critical value

$\sigma_{Y}^{\text {crit }}=\frac{B \phi}{100(1-\phi)}=\frac{l^{3} \rho_{0} c_{F} \phi}{100(1-\phi) t_{c}^{2} \sqrt{k}}$.

Hence, if all rock and experimental properties are fixed and sample length is increased, this critical value will increase until it is bigger than the actual value of $\sigma_{Y}$ for a given rock sample, and hence for longer samples the simpler fragmentation criterion will hold, $p_{0}>p_{0}^{\text {crit }}=\sigma_{Y}(1$ $-\phi)$, that is, the criterion is then independent of sample size. A typical (critical) value of $l$ for which the switch occurs (using typical values of the other parameters, as tabulated in Tables 1 and 2), is $20 \mathrm{~mm}$ with a range from 1 to $130 \mathrm{~mm}$.

For $l$ less than this critical value, the critical pressure for fragmentation to occur is

$p_{0}^{\text {crit }}=\frac{100 \sigma_{Y}^{2}(1-\phi)^{2} t_{c}^{2} \sqrt{k}}{l^{3} \rho_{0} c_{F} \phi}$

Clearly, this critical gas pressure for fragmentation reduces rapidly as sample length is increased, provided it remains below the critical length for switching mentioned above.

Fragment size, given that fragmentation occurs, is given by Eq. (22), and the dependence on sample length is not immediately clear from this complicated formula - the parameter $a$ depends on $l$. Hence we have graphed the value of fragment size versus sample length, for various values of permeability and porosity, in Figs. 20-23. In all of the cases considered, over the applicable range of lengths, fragment size (in $\mathrm{m}$ ) increases as sample length increases, according to our model. However, with the minor exception of the very low permeability case, the relative size of the fragment decreases with sample size.

\section{Conclusions}

The new model developed by Fowler et al. (2009) for the fragmentation of solid magma in shock-tube experiments has here been explored approximately and numerically and compared with experimental data. Model predictions of critical initial pressures versus porosity for fragmentation give good matches with data. Model predictions of fragmentation

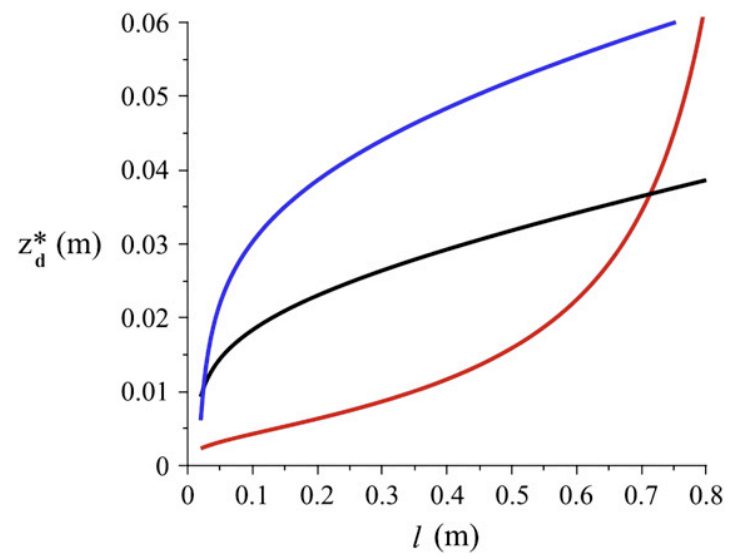

Fig. 20. The dependence of (dimensional) fragment size $z_{d}^{*}$ on sample length, for typical values of other parameters, except that permeability takes the values $10^{-10}$ (uppermost curve), $10^{-12}$ (middle curve), and $10^{-18}$ (lowermost curve).

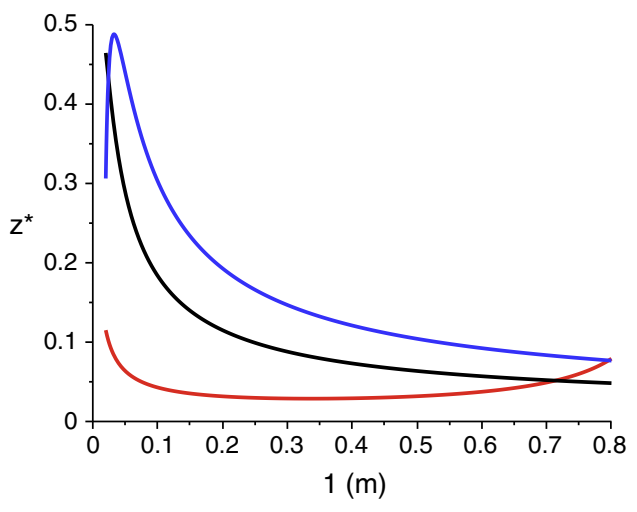

Fig. 21. The dependence of nondimensional fragment size $z^{*}$ as a fraction of sample length, on sample length, for typical values of other parameters, except that permeability takes the values $10^{-10}$ (uppermost curve), $10^{-12}$ (middle curve), and $10^{-18}$ (lowermost curve).

velocities versus initial gas pressure also lead to good matches with data over a wide range of porosities.

The model criterion for fragmentation, that the solid strain exceed the critical value $w_{z}^{\text {crit }}=(1-\phi) \sigma_{Y} / p_{0}$, together with a linear approximation to numerical results for solid strain, gives the approximation for sample tensile strength

$\sigma_{Y}=\frac{p_{0}^{\text {crit }}}{1-\phi}$

where $p_{0}^{\text {crit }}$ is the critical gas pressure for fragmentation of the sample. This approximation applies to porosities above $10 \%$, and is more accurate at higher porosities. It may provide an alternative method for measuring the tensile strength of rock, given its porosity.

The central role played by the relative speed of decompression at the surface of the sample is reflected by the importance of the parameter $a$ in our model. It appears in approximations based on our model and numerical solutions expressed in Eq. (22) for fragment size, and Eq. (24) for fragmentation velocity.

The temperature of rock samples has not been discussed previously in this paper, although hot samples have been distinguished from cold samples in Figs. 4 and 9. Hot samples (squares) are seen in these figures to fragment at relatively lower initial gas pressures than cold samples (circles), and we make a brief comment on this here. Temperature increases affect our model in several respects gas density and sample tensile strength are reduced; and the thermal expansion of the sample container compared to that of the rock sample is not a consideration in our work here (this could alter the initial stress state); glue strength will also be affected. The corresponding decrease in gas density implies a small decrease in the parameter $a$, which implies a slightly decreased maximum stress exerted by gas pressure differences on the solid. This increases by a small amount the critical gas pressure for fragmentation, against the trend seen in the data. The observed trend to a decreased critical pressure is consistent with a reduction in tensile strength with increasing temperature that overwhelms the density effect. It would be interesting to explore this further experimentally, especially for temperatures near the glass transition.

We found that in general, fragmentation occurs more readily for longer samples, up to a critical length where fragmentation becomes independent of sample size (and permeability and density). Fragment size generally increases with sample size.

Note that while it is tempting to conclude that very long gassy rock samples, such as might be found ex vitro, fragment independently of length, our conclusions do not extend beyond sample lengths of about $4 \mathrm{~m}$ for typical values of other parameters. This is partly because if sample length is increased further, the Reynolds number will then become small, 


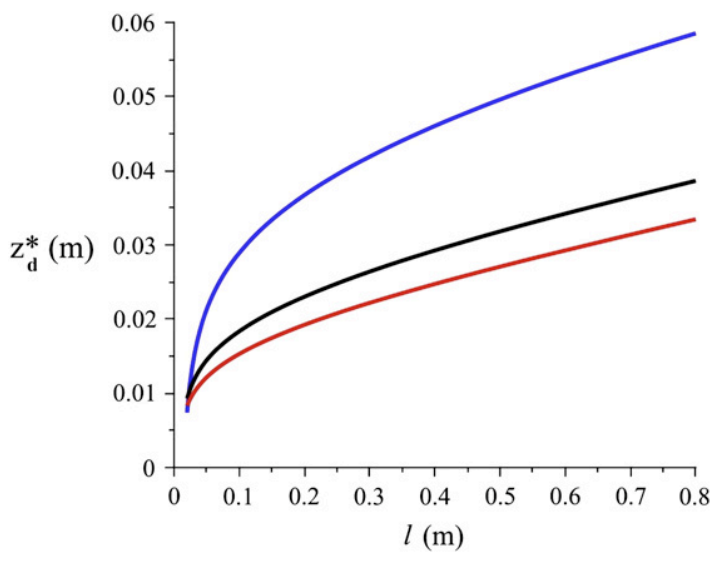

Fig. 22. The dependence of (dimensional) fragment size $z_{d}^{*}$ on sample length, for typical values of other parameters, except that porosity takes the values 0.05 (uppermost curve), 0.4 (middle curve), and 0.9 (lowermost curve).

and we will no longer be able to discard certain terms in the full model, and reduce it to the model we are solving here. So any reduced model for significantly longer length scales will be different. Another indication that it is unwise to try to extend length scales much beyond our laboratory values, is that the denominator of the fitted behaviour Eq. (22) goes through zero at higher sample lengths - the behaviour only fits over the given range of values of the parameter $a$.

So no firm conclusions can be drawn from our work, about length scales for volcanic applications ex vitro. This is not the only reason our model does not extend to volcanism in nature - our model was constructed specifically and exclusively to explain results of laboratory experiments on gas-charged solid porous rock samples, and in this respect it has been successful. However, modelling gas exsolution in a rising molten magma that is solidifying remains an interesting challenge and an outstanding problem.

\section{Acknowledgements}

M. J. M. is grateful for support provided by the Mathematics Applications Consortium for Science and Industry (MACSI, www.macsi.ul. ie), Department of Mathematics and Statistics, University of Limerick, while on Study Leave there. A. C. F. acknowledges the support of MACSI, funded by the Science Foundation Ireland Mathematics Initiative Grant 06/MI/005.

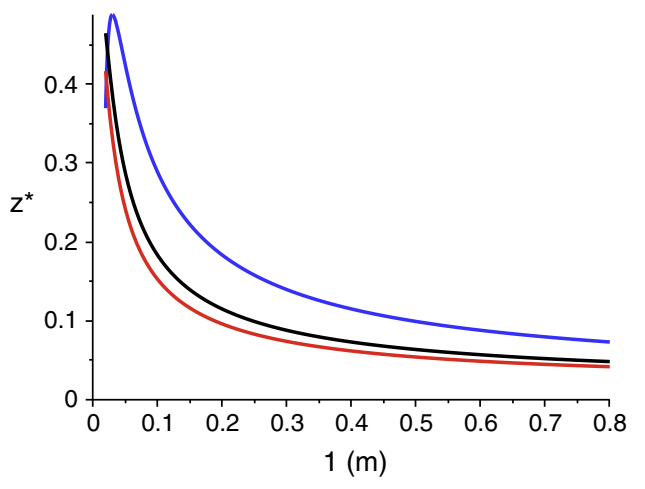

Fig. 23. The dependence of fragment size as a fraction of sample length, $z^{*}$ on sample length, for typical values of other parameters, except that porosity takes the values 0.05 (uppermost curve), 0.4 (middle curve), and 0.9 (lowermost curve).

\section{Appendix A. Dimensional model equations}

Here, for completeness, we summarise part of the dimensional model derived in Fowler et al. (2009) for the flow of gas out of a pressurised column of rock, in one dimension. Momentum conservation for the rock gives

$\rho_{s}(1-\phi) w_{t t}=(1-\phi) \sigma_{z}+A+D-F$,

where $w$ is vertical rock displacement $(\mathrm{m}), z$ is the vertical coordinate $(\mathrm{m}), \phi$ is rock porosity, $\rho_{s}$ is rock density $\left(\mathrm{kg} / \mathrm{m}^{3}\right), \sigma$ is the vertical component of stress in the rock ( $\mathrm{Pa}$ ), a $t$ subscript means a time derivative, a $t t$ subscript means the second time derivative, and a $z$ subscript means the z-derivative of the variable. Momentum conservation in the gas gives

$\rho_{f} \phi v_{t}=-\phi p_{z}-A-D$,

where $\rho_{f}$ is gas density, $v$ is gas velocity, and hence $v_{t}$ is gas acceleration. Stress and strain are related by

$(1-\phi) \sigma=E w_{z}-\alpha p$

where $E$ is an elastic constant, typically with a value of about $10^{11} \mathrm{~Pa}$ for rock, and the dimensionless parameter $\alpha$ relates to the deformability of the pore space and the gas and is presumed of order one. The term $A$ accounts for an added mass effect, and corresponds physically to the concept that moving a piece of rock past gas requires displacing the gas backwards (Biot, 1956), and can be written

$A=(1-\phi) C_{V M} \rho_{f}\left(v_{t}-w_{t t}\right)$,

where $C_{V M}$ is an order one constant relating the added mass density to the porosity and gas density. The term $D$ has dimensions of pressure gradient and accounts for the interfacial drag when gas moves past rock, and when Darcy's law for flow in a porous medium is extended to Forchheimer's equation to include turbulent flow effects, takes the form

$D=\frac{\eta_{f} \phi^{2}}{k}\left(v-w_{t}\right)+\frac{\rho_{f} C_{F} \phi^{3}}{\sqrt{k}}\left(v-w_{t}\right)\left|v-w_{t}\right|$,

where $\eta_{f}$ is the dynamic viscosity of the gas (about $18 \times 10^{-6}$ Pa.s), $k$ is the permeability of the rock (about $10^{-12} \mathrm{~m}^{2}$ ), and $C_{F}$ is the dimensionless Ergun coefficient (about 0.5). Conservation of gas mass gives

$\left(\rho_{f} \phi\right)_{t}+\left(\rho_{f} \phi v\right)_{z}=0$

and assuming adiabatic expansion of the gas, we can relate gas pressure and density as

$\rho_{f}=\rho_{0}\left(\frac{p}{p_{0}}\right)^{\frac{1}{\gamma}}$

where $\gamma$ is the adiabatic index, with value 1.4 for a diatomic ideal gas like the nitrogen used in many of the shock tube experiments, and $\rho_{0} \approx 115 \mathrm{~kg} \cdot \mathrm{m}^{-3}$ and $p_{0} \approx 10 \mathrm{MPa}$ are representative values of gas density and pressure. Wall "friction" due to the glue that temporarily holds the sample down is expressed as

$F=\frac{\mu_{g}\left(w-w_{0}\right)}{2 \pi R d_{g}}$,

where $\mu_{\mathrm{g}} \approx 10^{9} \mathrm{~Pa}$ is the glue shear modulus, $R=1.2 \mathrm{~cm}$ is the radius of the sample, and $d_{g} \approx 0.8 \mathrm{~mm}$ is the thickness of the glue. $w_{0}(z)$ is the initial displacement of the sample, after glueing the sample and pressurising the chamber. Note that this equation relates shear force and shearing 
displacement at the wall, due to the presence of glue there. Without the glue, the rock sample simply takes off like a gas-driven projectile when gas pressure is released. Values of physical constants are summarised in Table 2, and those properties with significant ranges of values are listed in Table 1.

The criterion for fracturing of rock is taken to be that the effective stress $(1-\phi)(\sigma+p)$ exceeds a yield stress $(1-\phi) \sigma_{Y}$, that is, that

$\sigma+p>\sigma_{Y}$

where $\sigma_{Y}$ is the tensile strength of the rock sample, with dependence on porosity removed (that is, for low porosity samples), typically about $2 \mathrm{MPa}$.

Initial conditions for these coupled differential and algebraic equations are zero gas and solid velocities, and some small initial displacement $w_{0}(z)$ due to slowly pressurising the sample, and stress given by the chamber pressure $p_{0}$ after charging,

$w_{t}=v=0, \quad-\sigma=p=p_{0}, \quad t=0$.

Boundary conditions are zero displacement and gas flow at the glued base, and a specified (measured) time-varying gas pressure $p_{c}(t)$ at the upper surface $z=l$ of the sample as determined by the release mechanism,

$v=w=0 \quad$ at $\quad z=0, \quad-\sigma=p=p_{c}(t)$ at $\quad z=l$,

where $p_{c}$ is taken directly from experimental measurement of gas pressure in the chamber just above the sample, and is found to take the form

$p_{c}=e^{-\frac{t}{t_{c}}}$

where $t_{c}$ is the shock chamber relaxation time, typically about $1 \mathrm{~ms}$

\section{Appendix B. Nondimensional model equations}

The dimensional model equations are rescaled and nondimensionalised to variables with a tilde on top, by the transformations

$$
\begin{array}{lrrl}
\rho_{f}=\rho_{0} \tilde{\rho}_{f}, & \sigma=p_{0} \tilde{\sigma}, & p=p_{0} \tilde{p} \\
z=l \tilde{z}, & t=t_{0} \tilde{t}, & t_{0}=\frac{l}{v_{0}} \\
w=\bar{w} \tilde{w}, & \bar{w}=\frac{p_{0} l}{E}, & v=v_{0} \tilde{v} \\
v_{0}=\left(\frac{\sqrt{k} p_{0}}{\phi \rho_{0} C_{F} l}\right)^{\frac{1}{2}} \approx 0.85 \mathrm{~ms}, & D=\left(\frac{\phi p_{0}}{l}\right) \tilde{D} & A=\left(\frac{\rho_{0} v_{0}^{2}}{l}\right) \tilde{A}
\end{array}
$$

Note that the time and length-scales chosen correspond to gas velocities well below the speed of sound in the gas.

The resulting dimensionless equations are

$$
\begin{aligned}
& \epsilon(1-\phi) \tilde{\mathrm{w}}_{t t}=(1-\phi) \tilde{\sigma}_{z}+v \tilde{A}+\tilde{D}-\lambda\left(\tilde{w}-\tilde{w}_{0}\right) \\
& v \phi \tilde{p}^{\frac{1}{\gamma}} \tilde{v}_{t}=-\phi \tilde{p}_{\tilde{z}}-v \tilde{A}-\tilde{D} \\
& (1-\phi) \tilde{\sigma}=\tilde{w}_{\tilde{z}}-\alpha \tilde{p} \\
& \tilde{A}=(1-\phi) C_{V M} \tilde{p}^{\frac{1}{\gamma}}\left(\tilde{v}_{\tilde{t}}-\delta \tilde{w}_{\tilde{t} \tilde{t}}\right) \\
& \tilde{D}=\left(\frac{\tilde{v}-\delta \tilde{w}_{\tilde{t}}}{\operatorname{Re}_{p} C_{F}}\right)+\phi \tilde{p}^{\frac{1}{\gamma}}\left(\tilde{v}-\delta \tilde{w}_{\tilde{t}}\right)\left|\tilde{v}-\delta \tilde{w}_{\tilde{t}}\right| \\
& \left(\tilde{p}^{\frac{1}{\gamma}}\right)_{\tilde{t}}+\left(\tilde{p}^{\frac{1}{\gamma}} \tilde{v}\right) \tilde{z}=0
\end{aligned}
$$

with dimensionless pressure in the chamber above the sample satisfying

$\tilde{p}_{c}=\exp (-a \tilde{t})$

and where parameters and values are

$$
\begin{array}{ll}
\epsilon=\frac{\rho_{s} v_{0}^{2}}{E} \approx 1.9 \times 10^{-7}, & v=\frac{\rho_{0} v_{0}^{2}}{p_{0}} \approx 0.8 \times 10^{-4}, \\
\lambda=\frac{\mu_{g} l^{2}}{2 \pi R d_{g} E} \approx 0.6, & \delta=\frac{p_{0}}{E} \approx 10^{-4}, \\
\operatorname{Re}_{p}=\frac{\rho_{0} v_{0} \sqrt{k}}{\eta_{f}} \approx 18, & a=\frac{t_{0}}{t_{c}} \approx 22,
\end{array}
$$

The usual convention is now to drop the tildes, so that unless otherwise stated, variables are dimensionless from now on.

\section{Appendix B.1. Reduced equations}

This complicated system of coupled equations can be reduced to a simpler system by setting the small parameters , $\delta, 1 / \operatorname{Re}_{p}$, and $v$ to zero, essentially obtaining the leading term in an asymptotic series expansion in these small parameters.

The resulting dimensionless system, dropping the tilde signs, using $p_{z}=-p^{\frac{1}{\gamma}}|v| v$ (obtained by combining Eqs. (B.3) and (B.6)), and setting $\phi+\alpha$ to one for simplicity since it is order one, is

$\frac{\partial p^{\frac{1}{\gamma}}}{\partial t}=\frac{\partial}{\partial z}\left(\left|p^{\frac{1}{\gamma}}\right|^{\frac{1}{2}} p_{z}\right)$,

$w_{z z}=p_{z}+\lambda w$,

with initial conditions

$w=w_{0}=0, \quad w_{t}=v=0, \quad-\sigma=p=1$ at $t=0$

and boundary conditions

$p_{z}=w=0$ at $z=0, \quad p=p_{c}=\exp (-a t), \quad w_{z}=0$ at $z=1$.

That is, pressure obeys the nonlinear diffusion Eq. (B.9), so that the pressure drop at the sample surface diffuses inwards, and displacement in the rock is then obtained by solving the boundary value problem Eq. (B.10). Note that the diffusion equation can be solved independently of rock displacement.

These equations apply right up to the time that the rock first ruptures, the time of primary fragmentation. This occurs when the effective stress exceeds the critical value, $p+\sigma>\sigma_{Y}$. Noting that $\alpha \approx 1-\phi$, Eq. (B.4) gives

$p+\sigma=\frac{w_{z}}{1-\phi}$.

Hence the criterion for fragmentation can be written in the form

$w_{z}>(1-\phi) \sigma_{Y}$

where $\sigma_{Y}$ is nondimensional tensile strength, approximately 0.2 in value if $p_{0}$ is $10 \mathrm{MPa}$ and dimensional tensile strength is $2 \mathrm{MPa}$.

So the rock fragmentation problem has been reduced to first solving the nonlinear diffusion problem (Eq. (B.9)) for $p$, while at the same time computing $w$ by using the solution $p$ in the boundaryvalue problem (Eq. (B.10)). Then at each time, the criterion Eq. (B.11) is checked, until primary fragmentation occurs. 


\section{Appendix C. Green's function for strain}

The Green's function $G\left(z, z_{0}\right)$ for solving

$w_{z z}-\lambda w=p_{z}$

with $p_{z}$ prescribed as a function of $z$ at a given time, and boundary conditions $w(0)=0, w_{z}(1)=0$, satisfies

$G_{z z}-\lambda G=\delta_{D}\left(z-z_{0}\right)$

where $\delta_{D}$ is the Dirac delta function, with the usual continuity conditions across the jump at $z=z_{0}$. $G$ is given by the formula

$G\left(z, z_{0}\right)=-\frac{1}{v D} \begin{cases}\sinh (v z) \cosh \left(v\left(1-z_{0}\right)\right), & z \leq z_{0} \\ \sinh \left(v z_{0}\right) \cosh (v(1-z)), & z>z_{0}\end{cases}$

where $d=\cosh v$ and $v=\sqrt{\lambda}$.

Then $w_{z}$ is obtained by the quadrature

$w_{z}(z)=\int_{0}^{1} G_{z}\left(z, z_{0}\right) p_{z}\left(z_{0}\right) d z_{0}$,

where the derivative of the Green's function is

$G_{z}\left(z, z_{0}\right)=-\frac{1}{D}\left\{\begin{array}{cc}\cosh (v z) \cosh \left(v\left(1-z_{0}\right)\right), & z \leq z_{0} \\ -\sinh \left(v z_{0}\right) \sinh (v(1-z)), & z>z_{0}\end{array}\right.$

This formula has been tested in Matlab by comparing with a direct numerical solution to Eq. (B.10) using the routine bvp $4 \mathrm{c}$.

\section{Appendix D. Lumped parameter model}

The average gas pressure in the rock sample may be approximated by noting that the gas pressure satisfies

$\frac{\partial p^{\frac{1}{\gamma}}}{\partial t}=-\frac{\partial}{\partial z}\left(v p^{\frac{1}{\gamma}}\right)$

where

$v=\sqrt{\frac{-p_{z}}{p^{\frac{1}{\gamma}}}}$

We approximate the pressure difference driving gas flow by the average pressure $\hat{p}$ minus the surface pressure $p_{c}$ which we set to zero, so that the pressure gradient is approximated by $p_{z} \approx-\hat{p} /(0.5)=-2 \hat{p}$. Then

$v \approx \sqrt{2} p^{\frac{\gamma-1}{2 \gamma}}$

Our gas diffusion equation becomes, after a further replacement of $p_{z}$,

$\frac{\partial \hat{p}^{\frac{1}{\gamma}}}{\partial t} \approx-\sqrt{2}\left(\frac{\gamma+1}{\gamma}\right) \hat{p}^{\frac{\gamma+1}{2 \gamma}}$

This integrates to give

$\hat{p} \approx \frac{1}{\left(c_{1} t+1\right)^{c_{2}}}, \quad c_{1} \equiv \frac{\gamma^{2}-1}{\sqrt{2} \gamma}, \quad c_{2} \equiv \frac{2 \gamma}{\gamma-1}$

\section{Appendix E. Similarity solution}

An approximate formula for pore gas pressure, based on solving Eq. (B.9) for small times, assuming the surface pressure $p_{c}$ changes relatively slowly, can be obtained in the form of the similarity solution

$p \approx R(\eta)^{\gamma}, \quad \eta=\frac{1-z}{B t^{2 / 3}}$,

where $R$ satisfies the differential equation

$\left(R^{\gamma} \sqrt{R^{\prime}}\right)^{\prime}+\eta R^{\prime}=0$

if we choose $B=(9 \gamma / 4)^{1 / 3}$. The boundary conditions for $R$ are

$R(0)=R_{0}=p_{c}^{1 / \gamma}, \quad R \rightarrow 1$ as $\eta \rightarrow \infty$.

\section{References}

Alidibirov, M., 1994. A model for viscous magma fragmentation during volcanic blasts. Bulletin of Volcanology 56, 459-465.

Alidibirov, M., Dingwell, D.B., 1996. Magma fragmentation by rapid decompression. Nature 380, 146-148.

Biot, M.A., 1956. Theory of propagation of elastic waves in a fluid-saturated porous solid. I. Low-frequency range. The Journal of the Acoustical Society of America $28,168-178$.

Biot, M.A., 1962. Mechanics of deformation and acoustic propagation in porous media. Journal of Applied Physics 33, 1,482-1,498.

Cashman, K.V., Sturtevant, B., Papale, P., Navon, O., 2000. Magmatic fragmentation. In: Sigurdsson, H. (Ed.), Encyclopedia of Volcanoes. Academic Press, San Diego, pp. 421-430.

Costa, A., 2006. Permeability-porosity relationship: a re-examination of the KozenyCarman equation based on a fractal pore-space geometry assumption. Geophysical Research Letters 33, L02318, http://dx.doi.org/10.1029/2005GL025134.

Fowler, A.C., Scheu, B., Lee, W.T., McGuinness, M.J., 2009. A theoretical model of the explosive fragmentation of vesicular magma. Proceedings of the Royal Society of London. Series A 466, 731-752, http://dx.doi.org/10.1098/rspa.2009.0382 (March 8, 2010, published online before print November 3, 2009).

Gilbert, J.S., Sparks, R.S.T. (Eds.), 1998. The Physics of Explosive Volcanic Eruptions. Geological Soc. Special Publications, 145. Geological Society, London, UK.

Heiken, G., Wohletz, K., 1991. Fragmentation processes in explosive volcanic eruptions. Sedimentation in volcanic settings: Society for Sedimentary Geology, Special Publications, 45 , pp. $19-26$

Ichihara, M., Rittel, D., Sturtevant, B., 2002. Fragmentation of a porous viscoelastic material: implications to magma fragmentation. Journal of Geophysical Research 107 (B10), 2229, http://dx.doi.org/10.1029/2001JB000591.

Kameda, M., Kuribara, H., Ichihara, M., 2008. Dominant time scale for brittle fragmentation of vesicular magma by decompression. Geophysical Research Letters 35 L14302, http://dx.doi.org/10.1029/2008GL034530.

Kennedy, B., Spieler, O., Scheu, B., Kueppers, U., Taddeucci, J., Dingwell, D.B., 2005. Conduit implosion during Vulcanian eruptions. Geology 33 (7), 581-584.

Koyaguchi, T., Mitani, N.K., 2005. A theoretical model for fragmentation of viscous bubbly magmas in shock tubes. Journal of Geophysical Research 110 (B10), B10202, http://dx.doi.org/10.1029/2004JB003513.

Koyaguchi, T., Scheu, B., Mitani, N.K., Melnik, O., 2008. A fragmentation criterion for highly viscous bubbly magmas estimated from shock tube experiments. Journal of Volcanology and Geothermal Research 178, 58-71.

Mader, H.M., Zhang, Y., Phillips, J.C., Sparks, R.S.J., Sturtevant, B., Stolper, E., 1994. Experimental simulations of explosive degassing of magma. Nature 372, 85-88.

Mangan, M.T., Cashman, K.V., 1996. The structure of basaltic scoria and reticulite and inferences for vesiculation, foam formation, and fragmentation in lava fountains. Journal of Volcanology and Geothermal Research 73, 1-18.

Martel, C., Dingwell, D.B., Spieler, O., Pichavant, M., Wilke, M., 2000. Fragmentation of foamed silicic melts: an experimental study. Earth and Planetary Science Letters $178,47-58$.

McBirney, A.R., Murase, T., 1970. Factors governing the formation of pyroclastic rocks. Bulletin of Volcanology 34, 372-384.

Mueller, S., Scheu, B., Spieler, O., Dingwell, D.B., 2008. Permeability control on magma fragmentation. Geology V36 (5), 399-402, http://dx.doi.org/101130/G24605A.

Namiki, A., Manga, M., 2005. Response of a bubble bearing viscoelastic fluid to rapid decompression: implications for explosive volcanic eruptions. Earth and Planetary Science Letters 236, 269-284.

Phillips, J.C., Lane, S.J., Lejeune, A.M., Hilton, M., 1995. Gum rosin-acetone system as an analogue to the degassing behaviour of hydrated magmas. Bulletin of Volcanology 57, 263-268.

Scheu, B., 2005. Understanding silicic volcanism: constraints from elasticity and failure of vesicular magma. Ph.D. thesis, Munich University.

Scheu, B., Spieler, O., Dingwell, D.B., 2006. Dynamics of explosive volcanism at Unzen volcano: an experimental contribution. Bulletin of Volcanology 69 (2), 175-187.

Scheu, B., Ichihara, M., Spieler, O., Dingwell, D.B., 2008. A closer look at magmatic fragmentation. Geophysical Research Abstracts 10 (EGU2008-A-04786).

Sparks, R.S.J., 1978. The dynamics of bubble formation and growth in magmas: a review and analysis. Journal of Volcanology and Geothermal Research 3, 1-37. 
Spieler, O., Dingwell, D.B., Alidibirov, M., 2004a. Magma fragmentation speed: an experimental determination. Journal of Volcanology and Geothermal Research 129 109-123.

Spieler, O., Kennedy, B., Kueppers, U., Dingwell, D.B., Scheu, B., Taddeucci, J., 2004b. The fragmentation threshold of pyroclastic rocks. Earth and Planetary Science Letters 226, 139-148.

Verhoogen, J., 1951. Mechanics of ash formation. American Journal of Science 249, 729-739.
Wilson, L., Sparks, R.S.J., Walker, G.P.L., 1980. Explosive volcanic eruptions: IV. The control of magma properties and conduit geometry on eruption column behaviour. Geophysical Journal of the Royal Astronomical Society 63, 117-148.

Yokoo, A., Ishihara, K., 2006. Pressure waves derived from eruption movie images of Sakurajima Volcano. Annuals of Disaster Prevention Research Institute, Kyoto University 49 C, 219-224.

Zhang, Y., 1999. A criterion for the fragmentation of bubbly magma based on brittle failure theory. Nature $402,648-650$ 